\title{
Systematic Analysis of the Literature in Search of Defining Systemic Sclerosis Subsets
}

\author{
Tatiana Nevskaya ${ }^{1}$, Janet E. Pope ${ }^{1}$ (D), Matthew A. Turk ${ }^{1}$, Jenny Shu ${ }^{1}$, April Marquardt ${ }^{2}$, \\ Frank van den Hoogen ${ }^{3}$, Dinesh Khanna ${ }^{2}$, Jaap Fransen ${ }^{4}$, Marco Matucci-Cerinic $^{5}$, Murray Baron ${ }^{6}$, \\ Christopher P. Denton ${ }^{7}$ (D), and Sindhu R. Johnson ${ }^{8}$ (D)
}

\begin{abstract}
Objective. Systemic sclerosis (SSc) is a multisystem disease with heterogeneity in presentation and prognosis. An international collaboration to develop new SSc subset criteria is underway. Our objectives were to identify systems of SSc subset classification and synthesize novel concepts to inform development of new criteria. Methods. Medline, Cochrane MEDLINE, the Cumulative Index to Nursing and Allied Health Literature, EMBASE, and Web of Science were searched from their inceptions to December 2019 for studies related to SSc subclassification, limited to humans and without language or sample size restrictions.

Results. Of 5686 citations, 102 studies reported original data on SSc subsets. Subset classification systems relied on extent of skin involvement and/or SSc-specific autoantibodies $(n=61)$, nailfold capillary patterns $(n=29)$, and molecular, genomic, and cellular patterns $(n=12)$. While some systems of subset classification confer prognostic value for clinical phenotype, severity, and mortality, only subsetting by gene expression signatures in tissue samples has been associated with response to therapy.

Conclusion. Subsetting on extent of skin involvement remains important. Novel disease attributes including SSc-specific autoantibodies, nailfold capillary patterns, and tissue gene expression signatures have been proposed as innovative means of SSc subsetting.
\end{abstract}

Key Indexing Terms: autoimmune diseases, scleroderma, systemic sclerosis

Systemic sclerosis (SSc) is a multisystem autoimmune rheumatic disease characterized by microvascular injury and accumulation

This work was supported by a grant from the Scleroderma Foundation and the World Scleroderma Foundation. SRJ is supported by a Canadian Institutes of Health Research New Investigator Award and the Gurmej Kaur Dhanda Scleroderma Research Award. DK was funded by the National Institutes of Health/National Institute of Arthritis and Musculoskeletal and Skin Diseases grant 5K24AR063120-07.

${ }^{I}$ T. Nevskaya, MD, PhD, J.E. Pope, MD, MPH, M.A. Turk, MSc, J. Shu, $M D, H B S c$, Schulich School of Medicine \& Dentistry, Western University, London, Ontario, Canada; ${ }^{2}$ A. Marquardt, DO, D. Khanna, MD, MS, University of Michigan, Ann Arbor, Michigan, USA; ${ }^{3}$ F. van den Hoogen, MD, PhD, St. Maartenskliniek and Radboud University Nijmegen Medical Centre, Nijmegen, the Netherlands; ${ }^{4}$ J. Fransen, MSc, PhD, Radboud University Nijmegen Medical Centre, Nijmegen, the Netherlands; ${ }^{5}$ M. Matucci-Cerinic, MD, PhD, Department of Experimental and Clinical Medicine \& Division of Rheumatology AOUC, Florence Italy University of Florence, Florence, Italy; ${ }^{6} \mathrm{M}$. Baron, MD, McGill University, Division Head Rheumatology, Jewish General Hospital, Montreal, Quebec, Canada; ${ }^{7}$ C.P. Denton, FRCP, PhD, University College London, Division of Medicine, London, UK; ${ }^{8}$ S.R. Johnson, MD, PhD, Toronto Scleroderma Program,

Toronto Western and Mount Sinai Hospitals, Department of Medicine, and Institute of Health Policy, Management and Evaluation, University of Toronto, Toronto, Ontario, Canada.

The authors declare no conflicts of interest relevant to this article. Address correspondence to Dr. S.R. Johnson, Toronto Scleroderma Program, Toronto Western Hospital, 399 Bathurst Street, Toronto, ON M5T 2S8, Canada.Email:Sindhu.Johnson@uhn.ca.

Accepted for publication April 26, 2021. of collagen in skin and other organs, such as the musculoskeletal system, lungs, kidneys, and gastrointestinal (GI) tract. ${ }^{1,2,3,4,5,6}$ SSc is associated with poorer patient outcomes and lower quality of life when compared to other rheumatic diseases. ${ }^{7}$ The 2013 American College of Rheumatology/European League Against Rheumatism (ACR/EULAR) classification criteria for SSc include skin thickening, fingertip lesions, abnormal nailfold capillaries, and the presence of SSc-related autoantibodies, but do not differentiate subsets of patients with SSc. ${ }^{8}$ Subclassification of SSc into a number of pathogenetically homogenous subsets with similar clinical manifestations and outcomes would help segregate clearly between prognostically distinct disease subgroups. Despite the complex multiorgan nature of SSc, the subsets are frequently defined as being limited cutaneous $(\mathrm{lcSSc})$ or diffuse cutaneous (dcSSc), based on the location of skin involvement. ${ }^{9}$ This classification system gives insight into disease progression; however, within lcSSc and dcSSc, the course of disease is highly variable between patients. ${ }^{10,11}$ With a more modern perspective, our understanding of SSc subsets is changing. A combination of multisystem involvement, antibody profiling, genetic markers, and differences in proteomics may play a role in prognosis and treatment options. ${ }^{12,13,14,15,16}$ Further defining subsets of patients with SSc may help to prognosticate, especially in early disease. ${ }^{17}$

An international collaboration to develop new criteria to subset SSc is underway. ${ }^{18}$ Current perceptions around SSc subset criteria were identified by leading international experts. In a survey of 30 SSc experts from 13 countries, 90\% of experts use $>2$ subsets for classifying and treating their patients. ${ }^{19}$ Concepts 
such as progression rates and likely organ involvement are considered for subsetting patients with SSc informally in clinical practice.

There is a need for criteria to identify subsets of patients with SSc for recruitment into clinical trials of novel therapeutic agents, to inform management, and for prognosis in clinical care. Previous attempts to outline SSc subset classification criteria have relied mainly on clinical manifestations. ${ }^{20}$ However, in recent years, novel disease attributes including autoantibody profiles, nailfold capillary patterns, and gene expression signatures have been proposed as means of subsetting. The objectives of this study were to identify existing systems of subset classification in SSc and to synthesize novel concepts in subsetting through a systematic review of the literature.

\section{METHODS}

Data sources and search strategy. A search of publications related to SSc and subsets was performed using Medline, Cochrane MEDLINE, the Cumulative Index to Nursing and Allied Health Literature, EMBASE, and Web of Science from their inceptions to December 2019 (for search strategy and key terms, see Supplementary Table 1, available with the online version of this article). The research question was, "What are the advantages and disadvantages of existing systems of subset classification in patients with systemic sclerosis?"

Searches were supplemented by hand searching the bibliographies of relevant articles (including citation searching). Studies were limited to humans, without language or sample size restrictions. Non-English-language articles were translated by native-language speakers or machine software. EndNoteX9 software (Clarivate) was used to check for duplications.

Studies were screened and excluded if they (1) reported localized scleroderma or scleroderma-like syndromes; (2) were abstracts, case reports, or review articles; or (3) were studies for which updated manuscripts were available. All articles were divided between 4 research groups (DK/CD, $\mathrm{JF} / \mathrm{FV}, \mathrm{MM} / \mathrm{JP} / \mathrm{JS} / \mathrm{TN}, \mathrm{MB} / \mathrm{SJ} / \mathrm{TN}$ ) and independently reviewed by investigators from each group using a standardized data abstraction form.
Abstracted data included classification schema, number of SSc subsets, number of subjects, country of origin, stated and perceived advantages and disadvantages of the classification system, and external validation. The systematic review conforms to the Preferred Reporting Items for Systematic Reviews and Meta-Analyses statement. The Strengthening the Reporting of Observational Studies in Epidemiology (STROBE) checklist was used to assess the reporting quality of the included studies.

\section{RESULTS}

Search results. Our literature review identified 5686 citations, of which 5584 were excluded because they were not relevant (conditions other than SSc, no classification system proposed), they had insufficient data, the data were not original, and/or they did not involve humans. The remaining 102 studies reported schema to subset patients with SSc (Figure 1).

SSc subset criteria. Subset classification systems have historically relied on clinical manifestations, most commonly extent of skin involvement ( $\mathrm{n}=20$; Table 1$), 9,10,11,21-37$ molecular, genomic, and cellular patterns $(\mathrm{n}=12$; Table 2$),{ }^{38-49}$ SSc-specific autoantibodies $(\mathrm{n}=46$, including 5 studies exploring both clinical and serological subsets ${ }^{10,21,27,29,37}$; Table 3), ${ }^{10,21,27,29,37,50-90}$ and abnormal nailfold capillary patterns $(\mathrm{n}=10$; Table 4).91-100 Twenty-one studies reporting associations between capillary abnormalities and clinical features or serology were included (Table 5).94,99,101-119 Using the STROBE checklist, the majority provided a clear presentation of what was planned, done, and found (Supplementary Table 2, available with the online version of this article). ${ }^{120}$

SSc subsets based on the extent of skin involvement. The diffuse vs limited SSc criteria of LeRoy, et al ${ }^{p}$ is the most commonly used system of SSc classification. The differences in development of visceral (renal and myocardial) disease and survival were shown for the subsets. ${ }^{911,25,26}$ The system has a good discriminative

7142 Medline, Embase, CINAHL, and Cochrane records

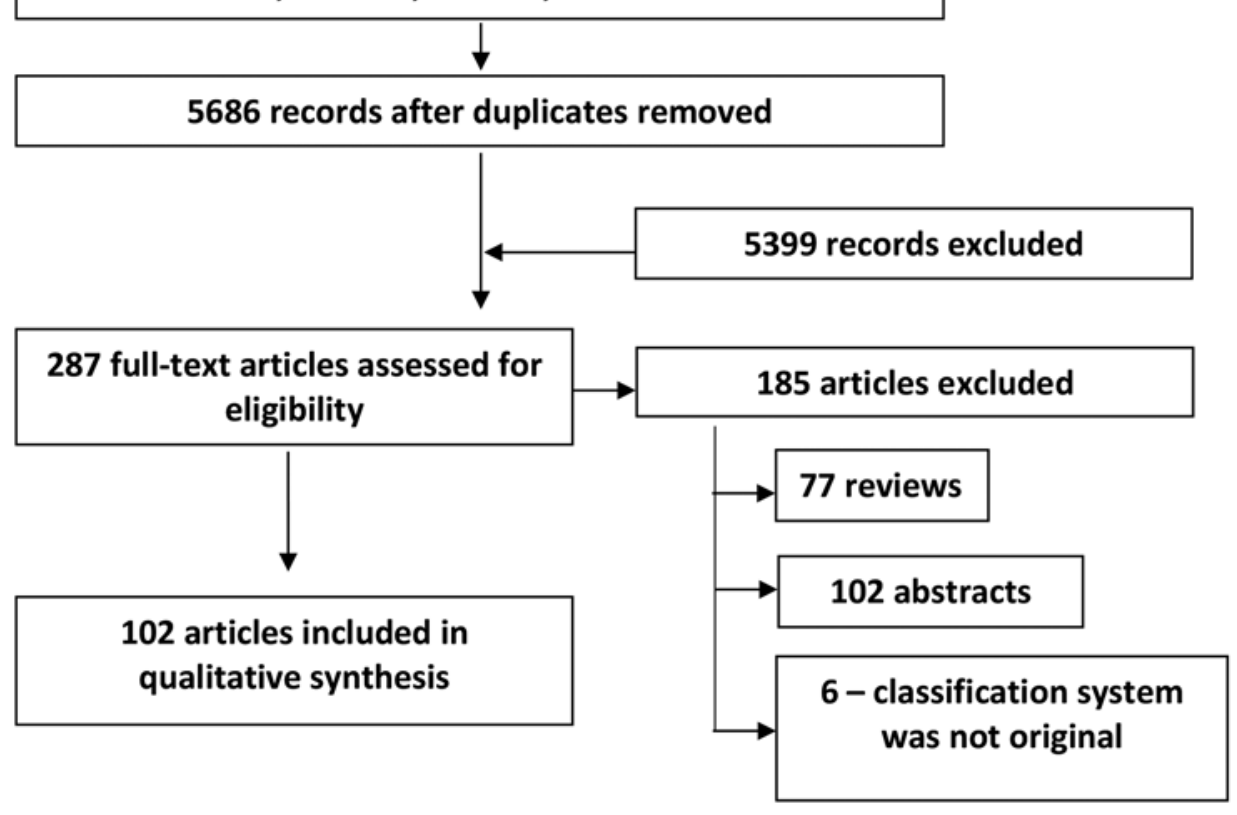

Figure 1. Flow diagram of search results. CINAHL: Cumulative Index to Nursing and Allied Health Literature. 
Table 1. Summary of clinical SSc subsets.

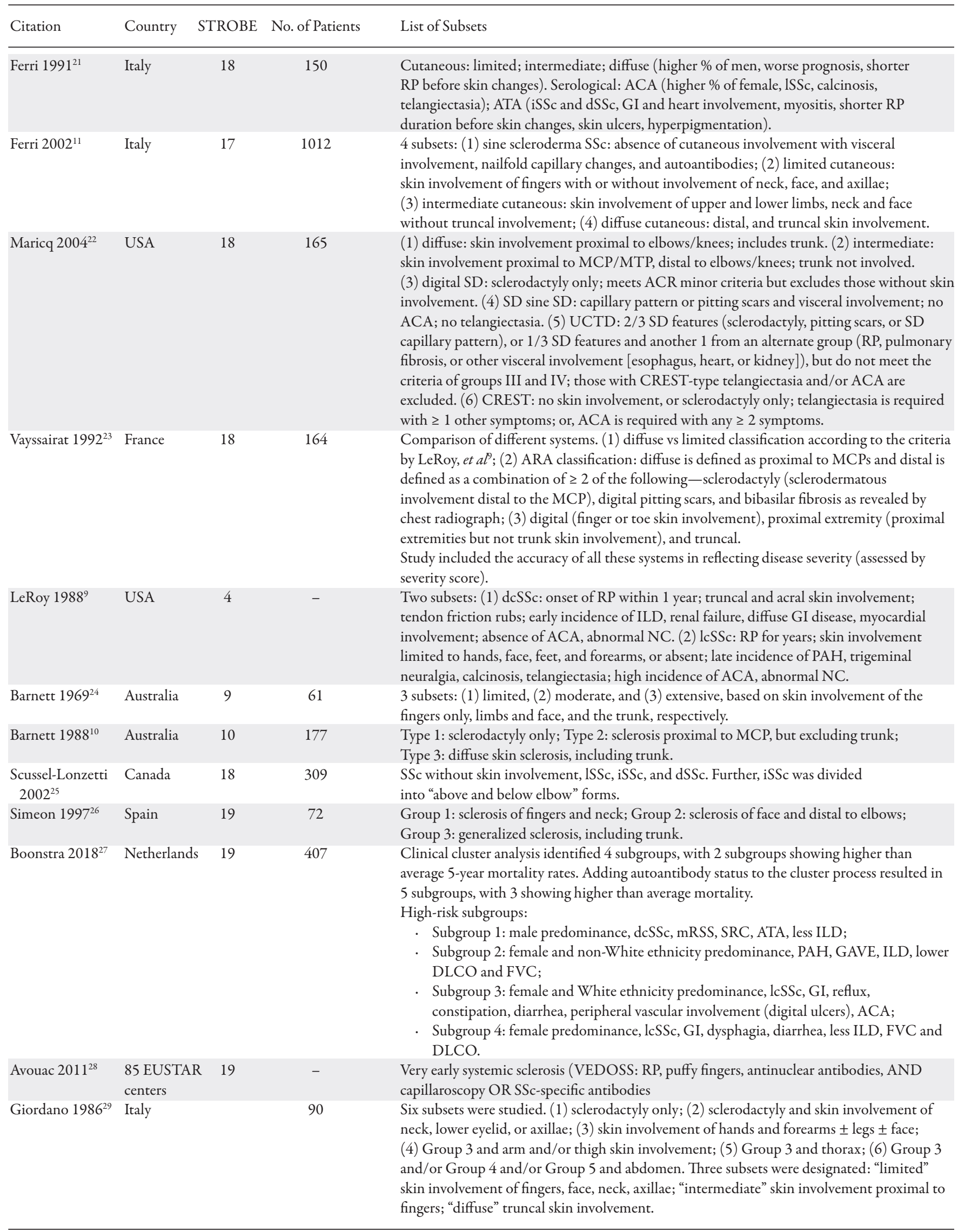


Table 1. Continued.

\begin{tabular}{|c|c|c|c|c|}
\hline Citation & Country & STROBE & No. of Patients & List of Subsets \\
\hline Goetz $1945^{30}$ & USA & 5 & 13 & $\begin{array}{l}\text { Two subsets: "acrosclerosis" and "diffuse", based on skin thickening limited to extremities or } \\
\text { includes trunk. }\end{array}$ \\
\hline LeRoy $2001^{32}$ & USA & 5 & - & $\begin{array}{l}\text { Four subsets: (1) ISSc consists of (a) objective RP AND any } 1 \text { of NC changes or SSc selective } \\
\text { autoantibodies OR (b) subjective RP AND both NC changes and SSc selective } \\
\text { autoantibodies; (2) lcSSc criteria for ISSc plus distal cutaneous changes; ( } 3 \text { ) dcSSc }{ }^{i} \text { criteria } \\
\text { for lcSSc plus proximal cutaneous changes; ( } 4 \text { ) diffuse fasciitis with eosinophilia: proximal } \\
\text { cutaneous changes without criteria for ISSc or lcSSc. }\end{array}$ \\
\hline Masi $1988^{33}$ & USA & 6 & - & $\begin{array}{l}\text { Three subsets: digital - skin involvement of fingers or toes but not proximal extremity or } \\
\text { trunk; proximal extremity - proximal extremities or face but not trunk; truncal - thorax or } \\
\text { abdomen. }\end{array}$ \\
\hline Rodnan $1979^{34}$ & USA & 6 & 273 & $\begin{array}{l}\text { Three subsets: (1) classical disease involving skin of the trunk, face, and proximal extremities, } \\
\text { as well as early involvement of esophagus, intestine, heart, lung, and kidney; (2) CREST } \\
\text { syndrome; and (3) overlap syndromes including sclerodermatomyositis and MCTD. }\end{array}$ \\
\hline Tuffanelli $1962^{36}$ & USA & 9 & 727 & $\begin{array}{l}\text { Two subsets: (1) acrosclerosis: RP, acral skin involvement; (2) dSSc: no RP, skin involvement } \\
\text { beginning centrally. }\end{array}$ \\
\hline Sobanski $2019^{37}$ & $\begin{array}{l}120 \text { EUSTAR } \\
\text { centers }\end{array}$ & R 19 & 6927 & $\begin{array}{l}\text { Two clusters: (1) lcSSc }(81 \%), 2 / 3 \text { without severe organ damage, ACA+ } \\
(54 \%) ;(2) \text { dcSSc }(61 \%) \text {, younger at disease onset, severe organ damage, ATA+ (54\%), } \\
\text { reduced survival. }\end{array}$ \\
\hline
\end{tabular}

ACA: anticentromere autoantibodies; ACR: American College of Rheumatology; ANA: antinuclear autoantibodies; ARA: American Rheumatism Association; ATA: antibodies to topoisomerase I; CREST: calcinosis, RP, esophageal involvement, sclerodactyly, telangiectasia; dcSSc: diffuse cutaneous SSc; DLCO: diffusing capacity for carbon monoxide; dSSc: diffuse SSc; GAVE: gastric antral vascular ectasia; EUSTAR: European Scleroderma Trials and Research; FVC: forced vital capacity; GI: gastrointestinal; ILD: interstitial lung disease; iSSc: intermediate SSc; lcSSc: limited cutaneous SSc; 1SSc: limited SSc; MCP: metacarpophalangeal joints; MCTD: mixed connective tissue disease; mRSS: modified Rodnan skin score; MTP: metatarsophalangeal joints; NC: nailfold capillaroscopy; PAH: pulmonary arterial hypertension; RP: Raynaud phenomenon; SD: scleroderma; SRC: scleroderma renal crisis; SSc: systemic sclerosis; STROBE: Strengthening the Reporting of Observational Studies in Epidemiology checklist; UCTD: undifferentiated connective tissue disorder; VEDOSS: very early diagnosis Of SSc.

value to identify the groups of patients with different dominant features (vascular vs fibrotic), internal organ damage, and outcome. It enables identification of patients with early SSc with poor prognosis who will need close monitoring and facilitates the comparison of more homogenous groups of patients in epidemiological studies and clinical trials. The LeRoy 1988 classification system ${ }^{9}$ has the advantage of comprising only 2 groups and requires criteria other than cutaneous involvement. To classify as diffuse SSc (dSSc), the prerequisites are the onset of Raynaud phenomenon (RP) within 1 year of the onset of skin involvement, early and significant visceral involvement, and the absence of anticentromere antibodies (ACA). When using these strict LeRoy criteria, dSSc represents only a small portion (8.5\%) of the total group with definite SSc. ${ }^{23}$ Two SSc-specific autoantibodies were included in the original LeRoy criteria: antitopoisomerase I antibodies (ATA) and ACA.

Acknowledging the important role of autoantibodies and capillary abnormalities, LeRoy updated the classification in 2001, proposing 4 subsets: limited SSc (ISSc), lcSSc, dcSSc, and diffuse fasciitis with eosinophilia. The classification includes ISSc as RP only in association with serological and/or capillary abnormalities. ${ }^{32}$ Considering that $\mathrm{SSc}$ is a multistage multiorgan disorder, ISSc is likely an early stage of disease and corresponds to very early SSc in the classification of Avouac, et al. ${ }^{28}$
Others have proposed 3 subset systems based on the extent of cutaneous involvement within the first year of presentation: type I digital (finger or toe skin involvement), type II intermediate (skin involvement proximal to metacarpophalangeal $[\mathrm{MCP}]$ joints, but excluding trunk), and type III diffuse (truncal sclerosis). ${ }^{10,24,29,33}$ The latter type was characterized by male predominance, shorter RP before skin changes, and worse prognosis. ${ }^{11}$ The clinical distinctiveness of the types was confirmed by difference in autoantibody profile: ACA was found more frequently in type I, while ATA was more frequent in intermediate SSc (iSSc) and dSSc. In the study, the authors included only SSc patients with disease duration $\leq 2$ years after the onset of skin lesions, and none of the patients had received any treatment that could potentially affect skin sclerosis prior to the enrollment. That ruled out the possibility that the iSSc group consisted of patients with SSc that would evolve into dSSc later or who originally had $\mathrm{dSS} c$ with skin regression under the treatment. Compared to the 2-subset LeRoy system, this classification better reflects the clinical heterogeneity of disease and identifies the subgroups with milder or more severe clinical prognostic evolution.

The simplicity of this 3-subset classification, which is based on clinical examination of skin only and does not require special equipment or tests, makes it highly reproducible and suitable for clinical care and research studies. Notably, this classification 
Table 2. Molecular, genomic, and cellular SSc subsets.

\begin{tabular}{|c|c|c|c|c|}
\hline Citation & Country & STROBE & No. of Patients & List of Subsets \\
\hline Milano $2008^{38}$ & USA & 21 & $\begin{array}{l}24 \text { SSc, } 3 \text { morphea, } \\
6 \text { healthy controls } \\
\text { (skin) }\end{array}$ & $\begin{array}{l}\text { Normal-like, diffuse proliferation, inflammatory, limited signatures. } \\
\text { Diffuse proliferation: higher mRSS, all dcSSc, longer disease duration } \\
\text { compared to patients with dcSSc in the inflammatory and normal-like groups; } \\
\text { increased number of proliferating cells in the epidermis. } \\
\text { - Inflammatory: both lcSSc and dcSSc; increased T cell infiltration in the dermis. } \\
\text { Limited: lcSSc, more severe RP. } \\
\text { Normal-like: both dcSSc and lcSSc. }\end{array}$ \\
\hline Mahoney $2015^{41}$ & USA & 22 & $\begin{array}{l}3 \text { SSc patient cohorts } \\
\text { from the studies } s^{37,38,39} \\
\text { (skin) }\end{array}$ & $\begin{array}{l}\text { Normal-like, fibroproliferative, inflammatory. } \\
\text { Identified the core sets of genes consistently associated with the intrinsic subsets, } \\
\text { and created a gene-gene interaction network across the intrinsic subsets. }\end{array}$ \\
\hline Taroni $2015^{42}$ & USA & 21 & $\begin{array}{l}16 \text { SSc, } 7 \text { controls } \\
\text { (esophageal biopsies) }\end{array}$ & $\begin{array}{l}\text { Inflammatory, noninflammatory, and proliferative. } \\
\text { - Independent of dcSSc/lcSSc subtypes, serum autoantibodies, and esophagitis. } \\
\text { - Inflammatory: older, a trend towards ILD (reduced DLCO, FVC, TLC). }\end{array}$ \\
\hline $\begin{array}{l}\text { Chakravarty } \\
2015^{43}\end{array}$ & USA & 22 & $\begin{array}{l}13 \text { SSc }(10 \text { treatment, } \\
3 \text { placebo), } 4 \text { healthy controls }\end{array}$ & $\begin{array}{l}\text { Fibroproliferative, inflammatory, and normal-like groups. } \\
\text {. } 4 / 5 \text { improvers mapped to the inflammatory intrinsic subset showed decreased } \\
\text { gene expression in inflammatory pathways over } 24 \text { weeks. One improver had } \\
\text { normal-like signature (spontaneous improver?). }\end{array}$ \\
\hline Gordon $2018^{44}$ & USA & 21 & $\begin{array}{l}15 \text { patients were assigned } \\
\text { to either an inflammatory } \\
\text { or a proliferative molecular } \\
\text { subset at baseline }\end{array}$ & $\begin{array}{l}\text { Inflammatory, proliferative, normal-like. } \\
\text { Molecular subset at baseline was not associated with clinical improvement } \\
\text { in the belimumab arm, the placebo arm, or the pooled treatment arms. } \\
\text { An overall reduction in inflammatory gene expression and movement toward } \\
\text { the normal-like subset was associated with improvement in mRSS; } 8 / 10 \\
\text { improvers were assigned to a normal-like molecular subset posttreatment. }\end{array}$ \\
\hline Frost $2019^{46}$ So & $\begin{array}{l}\text { outh Africa, } \\
\text { USA }\end{array}$ & 15 & 8 & $\begin{array}{l}\text { Two groups co-segregated with clinical features of ILD and/or inflammatory } \\
\text { myopathy, or the absence of an inflammation phenotype. These groups showed } \\
\text { paradoxical gene expression of the genes } T C F 7, S O X 17 \text {, and FRZB in affected and } \\
\text { unaffected skin. }\end{array}$ \\
\hline Franks $2019^{47}$ & USA & 21 & $\begin{array}{l}297 \text { skin biopsy samples } \\
\text { from } 102 \text { patients with }\end{array}$ & $\begin{array}{l}\text { Four intrinsic molecular subsets of SSc by supervised machine learning algorithms: } \\
\text { fibroproliferative, inflammatory, normal-like, and limited. }\end{array}$ \\
\hline
\end{tabular}

van der Kroef Netherlands,

$19 \quad 19$

19 Four clusters based on the distribution of monocyte subsets:

$2020^{48} \quad$ USA, Italy

- Cluster 1: high CD16+ monocytes and low memory B cell subsets, lcSSc;

- Cluster 2: increased classical monocytes, dcSSc, high mRSS, the strongest increase of CXCL10 and CXCL11 in the plasma;

- Cluster 3: larger amounts of memory B cells;

- Cluster 4: lower numbers of circulating classical monocytes, often no skin involvement.

$\begin{aligned} & \text { Martyanov } \\ & 2017^{49}\end{aligned}$
$\begin{aligned} & 20 \\ & 19 \text { patients with dcSSc } \\ & (12 \text { at baseline and }\end{aligned}$

- Skin-based intrinsic gene expression: fibroproliferative, inflammatory and normal-like.

posttreatment with dasatinib)

ABA: abatacept; dcSSc: diffuse cutaneous systemic sclerosis; DLCO: diffusing capacity for carbon monoxide; FVC: forced vital capacity; ILD: interstitial lung disease; IcSSc: limited cutaneous systemic sclerosis; MMF: mycophenolate mofetil; mRSS: modified Rodnan skin score; RP: Raynaud phenomenon; SSc: systemic sclerosis; STROBE: Strengthening the Reporting of Observational Studies in Epidemiology checklist; TLC: total lung capacity. 
Table 3. Associations between SSc-related autoantibodies and clinical SSc manifestations.

\begin{tabular}{llcl}
\hline Citation Country STROBE & $\begin{array}{c}\text { No. of } \\
\text { Patients }\end{array}$ & Autoantibodies Associations \\
\hline
\end{tabular}

\begin{tabular}{|c|c|c|c|c|}
\hline Barnett $1988^{10}$ & Australia & 10 & ACA & $\begin{array}{l}\text { SSc type: a higher frequency of ACA in type } 1 \text { SSc sclerodactyly only } \\
(60.8 \%) \text {, followed by type } 2 \text { sclerosis proximal to MCP, but excluding trunk } \\
(29.7 \%) \text {, and type } 3 \text { diffuse skin sclerosis including trunk }(9.5 \%) \text {. }\end{array}$ \\
\hline Ceribelli $2010^{50}$ & Italy, USA & 18 & anti-Th/To & $\begin{array}{l}\text { - lcSSc and mild slowly progressive ILD } \\
\text { higher frequency of pericarditis, male sex, lower FVC, younger patients } \\
\text { with SSc, and less frequent telangiectasia. }\end{array}$ \\
\hline Gliddon $2011^{51}$ & UK & 15 & $\begin{array}{c}\text { ACA, ATA, } \\
\text { anti-Th/To, } \\
\text { anti-RNAP I/II/III, } \\
\text { anti-U1-RNP, } \\
\text { unidentified ANA, } \\
\text { ANA-negative }\end{array}$ & $\begin{array}{l}\text { ACA: older at disease onset, isolated reduction in DLCO, reduced } \\
\text { creatinine clearance, telangiectasia, less frequent ILD } \\
\text { ATA: more extensive skin involvement, lung fibrosis } \\
\text { - Anti-U1-RNP: younger at disease onset, rare esophageal } \\
\text { involvement, less frequent telangiectasia }\end{array}$ \\
\hline Falkner $2000^{52}$ & USA & 19 & $\begin{array}{l}\text { ACA, ATA, } \\
\text { anti-Th/To, anti-RNAP III, } \\
\text { anti-fibrillarin, } \\
\text { unidentified ANA }\end{array}$ & $\mathrm{ACA}$ and anti-Th/To $-\mathrm{lcSSc}$ \\
\hline $\begin{array}{l}\text { Hamaguchi } \\
2008^{54}\end{array}$ & Japan & 20 & $\begin{array}{c}\text { ACA, ATA, } \\
\text { anti-U1-RNP, } \\
\text { anti-RNAP; Anti-Th/To } \\
\text { (small number of patients), } \\
\text { anti-U3-RNP (small number } \\
\text { of patients) }\end{array}$ & $\begin{array}{l}\text { - ATA: dcSSc, high mRSS, diffuse skin hyperpigmentation, pulmonary } \\
\text { fibrosis, decreased survival rate } \\
\text { - Anti-RNAP: dcSSc, high mRSS, finger contractures } \\
\text { - ACA: lcSSc, low mRSS, less frequent ILD } \\
\text { - Anti-U3-RNP: dcSSc, rarely decreased DLCO } \\
\text { - Anti-U1-RNP: low mRSS } \\
\text { - Anti-Th/To: low mRSS, rarely decreased DLCO and upper GI } \\
\text { involvement } \\
\text { - Negative ANA: low mRSS } \\
\text { - dcSSc-positive for anti-RNAP (compared to dcSSc-positive for ATA): } \\
\text { rapid skin progression, skin hyperpigmentation, less frequent pitting } \\
\text { scars and ILD, lower serum IgG levels }\end{array}$ \\
\hline Hanke $2010^{55}$ & Germany & 19 & $\begin{array}{l}\text { anti-CENP-A } \\
\text { or anti-CENP-B }\end{array}$ & $\begin{array}{l}\text { ACA (anti-CENP-A or anti-CENP-B): 1SSc; less frequent ILD, } \\
\text { cardiac involvement, skin ulcers }\end{array}$ \\
\hline Ferri $1991^{21}$ & Italy & 18 & ACA, ATA & $\begin{array}{l}\text { ACA: female predominance, lcSSc, calcinosis, telangiectasia } \\
\text { ATA: intermediate and diffuse SSc, GI and heart involvement, } \\
\text { myositis, skin ulcers, hyperpigmentation, shorter RP duration before } \\
\text { skin changes }\end{array}$ \\
\hline Harvey $1999^{56}$ & UK & 19 & $\begin{array}{c}\text { ACA, ATA, } \\
\text { anti-RNAP I/II/III }\end{array}$ & $\begin{array}{l}\text { ACA: IcSSc, rare renal disease and ILD } \\
\text { - ATA: ILD, renal involvement (compared to ACA) } \\
\text {. Anti-RNAP I/II/III: dcSSc }\end{array}$ \\
\hline $\begin{array}{l}\text { Hesselstrand } \\
2003^{57}\end{array}$ & Denmark & 19 & $\begin{array}{c}\text { ACA, ATA, } \\
\text { anti-RNAP I/II/III, } \\
\text { anti-U1-RNP, antihistone }\end{array}$ & $\begin{array}{l}\text { - ACA: less frequent ILD, female predominance, vascular changes } \\
\text { (finger systolic pressure), reduced GFR } \\
\text { - ATA: dSSc, higher \% of men, ILD } \\
\text { - anti-RNAP I/II/III: ILD } \\
\text { - anti-U1-RNP: younger at disease onset, vasospasm } \\
\text { - antihistone: more frequent cardiac, pulmonary and renal involvement, } \\
\text { reduced survival }\end{array}$ \\
\hline Song $2013^{58}$ & China, USA & 18 & $\begin{array}{l}\mathrm{ACA}^{*} \text { (anti-CENP-B } \\
\text { and anti-CENP-Q) }\end{array}$ & Less frequent ILD \\
\hline
\end{tabular}




Citation Country STROBE $\begin{gathered}\text { No. of } \\ \text { Patients }\end{gathered}$ Autoantibodies Associations

Hudson 2012 5 Canada $\quad 22 \quad 802 \quad$ ACA

\begin{tabular}{|c|c|c|c|c|}
\hline Kuwana $2005^{60}$ & Japan & 20 & 534 & Anti-RNAP III \\
\hline McCarty $1983^{61}$ & USA & 17 & $27 \mathrm{ACA}$ & $\mathrm{ACA}$ \\
\hline $\begin{array}{l}\text { Vázquez-Abad } \\
1994^{62}\end{array}$ & USA & 16 & 611 & ACA (CENP-B) \\
\hline Wu $2007^{63}$ & Israel, USA & 18 & $\begin{array}{l}50 \text { CREST } \\
21 \text { other }\end{array}$ & $\begin{array}{c}\text { Anti-CCP3 in } \\
\text { combination } \\
\text { with ACA }\end{array}$ \\
\hline Giordano $1986^{29}$ & Italy & 13 & 105 & $\mathrm{ACA}$ \\
\hline Santiago $2007^{64}$ & Canada & 19 & 242 & Anti-RNAP III \\
\hline Salazar $2015^{65}$ & USA & 19 & 3249 & ANA-negative \\
\hline Satoh $2009^{66}$ & Japan & 18 & 354 & Anti-RNAP III \\
\hline Sato $1998^{67}$ & Japan & 20 & 103 & $\begin{array}{l}\text { anticalpastatin } \\
\text { antibodies }\end{array}$ \\
\hline Simon $2009^{68}$ & Hungary & 19 & $\begin{array}{c}293 \text { (59 ATA } \\
\text { positive) }\end{array}$ & ATA fragment F1 \\
\hline $\begin{array}{l}\text { Iniesta Arandia } \\
2017^{69}\end{array}$ & Spain & 19 & 209 & $\begin{array}{l}\text { ACA, ATA and } \\
\text { anti-RNAP III- } \\
\text { positive }\end{array}$ \\
\hline $\begin{array}{l}\text { Boonstra } \\
2018^{27}\end{array}$ & Netherlands & 19 & 407 & $\begin{array}{l}5 \text { clusters based } \\
\text { on clinical and } \\
\text { serological features }\end{array}$ \\
\hline
\end{tabular}

$\begin{array}{ccccc}\text { Caetano } 2018^{70} & \text { UK } & 20 & 1313 & \begin{array}{c}\text { ACA+ dcSSc, } \\ \text { ACA+ lcSSc and } \\ \text { ACA-dcSSc } \\ \text { ACA, ATA, } \\ \text { Caramaschi } 2015^{71}\end{array} \\ & \text { Italy } & 5 & 178 & \begin{array}{c}\text { anti-RNAP III, } \\ \text { Th/To, PM/Scl }\end{array}\end{array}$

- ACA: older at disease onset, female predominance, lcSSc and lower mRSS, pulmonary hypertension, lower overall disease severity, less likely to have finger ulcers, digital tuft resorption, or finger contractures, ILD, SRC, inflammatory arthritis, and myositis.

- ACA status was predictive of the extent of skin involvement over time. Patients with lcSSc who were CENP-A-negative at baseline were more likely to progress to diffuse disease. dcSSc, higher maximum mRSS, and increased frequency of tendon friction rubs, SRC

Better prognosis, less frequent major renal, cardiac, pulmonary, and lower GI tract involvement compared to speckled or nucleolar ANA patterns CREST

CREST

- ACA: sclerodactyly with/without minimal skin involvement in other areas (armpits, eyelids, neck)

- ACA-negative (most were ATA-positive): arms, legs \pm trunk involvement, lower cumulative survival rate and higher severity of internal organ involvement

Risk of SRC

Less frequent vasculopathic manifestations

Severe skin and renal involvement

Higher ESR and inflammatory muscle involvement

No clinical associations

- ACA: female predominance, less common dcSSc and ILD, longer time from onset to SSc diagnosis

- ATA: higher prevalence of ILD, less frequent lcSSc and sine scleroderma subtypes

- Anti-RNAP III: dcSSc, malignancies more frequent, especially synchronous neoplasia

- No difference in terms of survival rate at 5 yrs and 30 yrs, or causes of death

- Autoantibodies improved detection of lung involvement, PAH and renal crisis, as well as patients with actual severe disease course, when shifting from clinical subgrouping to combined autoantibody and clinical subgrouping.

- High-risk (mortality around $10 \%)$ :

o Subgroup 1: dcSSc and renal crisis, lower female predominance, ATA+

o Subgroup 2: dcSSc, PAH, GAVE, less often White, ATA+, ACA-

- Intermediate (mortality risk $7.2 \%$ ):

o Subgroup 5: less frequent ILD and vasculopathy (pitting scars, digital ulcers), anti-RNAP III+, PM/Scl-

- Low risk:

o Subgroup 3: GI, ACA+, ATA-

o Subgroup 4: miscellaneous, PM/Scl+, RNAP-

$\mathrm{dcSS} c \mathrm{ACA}+$ : insidious onset of skin and major organ involvement, a lower incidence of ILD and SRC, and better survival than expected for dcSSc

- ACA: older patients, longer disease duration from RP onset

- ATA: ILD

- anti-RNAP III: SRC 
Table 3. Continued.

\begin{tabular}{|c|c|c|c|c|c|}
\hline Citation & Country ST & TROBE & $\begin{array}{l}\text { No. of } \\
\text { Patients }\end{array}$ & Autoantibodies & Associations \\
\hline Coppo $2013^{72}$ & France & $\begin{array}{r}1 \\
\text { in } \\
\text { var } \\
\text { dis } \\
\mathrm{n} \\
\text { dis } \\
\mathrm{n} \\
\mathrm{a} \\
\text { (G }\end{array}$ & \multicolumn{2}{|c|}{$\begin{array}{l}199 \text { individuals, } \\
\text { anti-HP1- } \\
\text { positive } \\
\text { suffering from } \\
\text { various autoimmune } \\
\text { disorders (Group I, } \\
\mathrm{n}=145 \text { ) and non } \\
\text { autoimmune } \\
\text { diseases (Group II, } \\
\mathrm{n}=44 \text { patients) } \\
\text { as well as healthy } \\
\text { individuals } \\
\text { (Group III, } \mathrm{n}=30 \text { ). }\end{array}$} & CREST \\
\hline $\begin{array}{l}\text { Foocharoen } \\
2017^{74}\end{array}$ & Thailand & 20 & $285 \quad$ Cl & $\begin{array}{l}\text { ATA, ACA } \\
\text { ENP A, CENP B), } \\
\text { anti-PM/Scl-100, } \\
\text { anti-PM/Scl-75, } \\
\text { nti-Ku, anti-Ro52, } \\
\text { anti-1RNAP III } \\
\text { RP11 and RP155), } \\
\text { anti-fibrillarin } \\
\text { RNP), anti-NOR-90, } \\
\text { Th/To, anti-PDGFR. }\end{array}$ & $\begin{array}{l}\text { ATA: female predominance, dcSSc, high peak mRSS, RP, hand } \\
\text { deformity } \\
\text {. ACA: negative association with hand deformity } \\
\text {. Anti-Ku: overlap syndrome SSc/PM }\end{array}$ \\
\hline $\begin{array}{l}\text { Hamaguchi } \\
2015^{75}\end{array}$ & Japan & 20 & 583 & Anti-RNAP III & $\begin{array}{l}\text { Anti-RNAP III: SRC, in particular, coexistence of anti-RNAP II and } \\
\text { anti-RNAP I/III (anti-RNAP I/II/III) and a higher ELISA index for } \\
\text { anti-RNAP III }\end{array}$ \\
\hline $\begin{array}{l}\text { Foocharoen } \\
2016^{77}\end{array}$ & Thailand & 17 & 294 & ATA, ACA & $\begin{array}{l}\cdot \text { ATA: hand deformity } \\
\text { - ACA: negative association with hand deformity } \\
\text { - } \text { ATA+dcSSc: earlier ILD vs ATA- } \\
\text {. } \text { ATA-lcSSc: RP }\end{array}$ \\
\hline $\begin{array}{r}\text { Hoa } 2016^{78} \text { Canac } \\
\text { US }\end{array}$ & $\begin{array}{l}\text { ada, Australia, } \\
\text { SA, Mexico }\end{array}$ & 20 & 2140 & anti-Ku & Anti-Ku: ILD, increased creatine kinase levels; no difference in survival \\
\hline Terras $2016^{79}$ & Germany & 16 & $158(11)$ & Anti-RNAP III & dcSSc, higher mRSS, renal involvement \\
\hline Perosa $2013^{80}$ & Italy & 21 & $\begin{array}{c}121 \text { (75 ACA } \\
\text { positive) }\end{array}$ & $\begin{array}{l}\text { ACA cross- } \\
\text { reacting with } \\
\text { FOXE3p } 53-62\end{array}$ & Less likely to develop active disease \\
\hline $\begin{array}{l}\text { Wodkowski } \\
2015^{90}\end{array}$ & $\begin{array}{l}\text { Canada, } \\
\text { ustralia, USA }\end{array}$ & 17 & 1574(103) an & $\begin{array}{l}\text { Monospecific } \\
\text { ti-Ro52/TRIM21 } \\
\text { antibodies }\end{array}$ & Less likely White, ILD, poor survival \\
\hline Shah $2010^{82}$ & USA & 19 & $23(6)$ & anti-RNAP I/III & Temporal relationship with the onset of cancer \\
\hline $\begin{array}{l}\text { Sánchez-Montalvá } \\
2014^{83}\end{array}$ & Spain & 19 & 132 & Anti-SSA/Ro52 & No clinical associations \\
\hline Shah $2019^{84}$ & USA & 18 & (su & $\begin{array}{l}\text { anti-RPA194 } \\
\text { ubgrouping among } \\
\text { anti-RPC155 } \\
\text { antibodies) }\end{array}$ & Cancer, less severe GI disease \\
\hline $\begin{array}{l}\text { Shayakhmetova } \\
2019^{85}\end{array}$ & Russia & 18 & $\begin{array}{l}330 \text { positive } \\
\text { for a-U1RNP }\end{array}$ & anti-U1RNP & $\begin{array}{l}\text { ISSc (91\%), digital ulcers/scars (50\%), ILD (63\%); often joint (65\%) } \\
\text { and muscle (43\%) involvement; } 1 / 3 \text { Sjogren syndrome }\end{array}$ \\
\hline
\end{tabular}




\begin{tabular}{|c|c|c|c|c|c|}
\hline Citation & Country $S$ & STROBH & $\begin{array}{c}\text { No. of } \\
\text { Patients }\end{array}$ & Autoantibodies & Associations \\
\hline Patterson $2015^{86}$ & 6 Australia & 18 & $\begin{array}{r}505 \\
\text { (we } \\
\text { anti } \\
\text { ant } \\
\text { an }\end{array}$ & $\begin{array}{l}\text { ACA, anti-RNAP III } \\
\text { (strong), anti-RNAP III } \\
\text { veak), ATA, anti-RNAP III, } \\
\text { ti-NOR-90, anti-fibrillarin, } \\
\text { nti-Th/To, anti-PM/Scl-75, } \\
\text { anti-PM/Scl-100, anti-Ku, } \\
\text { ATA, anti-Ro52, } \\
\text { anti-PDGFR }\end{array}$ & $\begin{array}{l}\text { - } 1 \text { SSc: ACA } \\
\text { - dcSSc: RNAP III, ATA } \\
\text {. Anti-Th/To: less likely joint contractures and reflux esophagitis } \\
\text {. Anti-fibrillarin: digital amputation and a trend toward GAVE } \\
\text { - Anti-TRIM-21/Ro 52: telangiectasia, dry eyes, PAH, and calcinosis } \\
\text { - Anti-PM/Scl-75/100: a history of digital ulcers and a trend toward } \\
\text { lcSSc, no history of smoking } \\
\text { - Anti-RNAP III: dcSSc, joint contractures, SRC; a strong RNAP III } \\
\text { cluster with increased risk of GAVE, lower risk of esophageal } \\
\text { dysmotility, shorter disease duration }\end{array}$ \\
\hline Perosa $2016^{87}$ & Italy & $21 \varepsilon$ & $\begin{array}{l}84 \text { anti-CENP } \\
\text { positive }\end{array}$ & $\begin{array}{l}\text { PA Subspecificities } \\
\text { of anti-CENPA: } \\
\text { anti-pc4. } 2 \text { antibodies, } \\
\text { anti-pc14.1 antibodies }\end{array}$ & $\begin{array}{l}\text { Anti-pc } 4.2 \text { antibodies: sPAP and inversely associated with DLCO } \\
\text { Anti-pc14.1 antibodies: inversely sPAP and positively DLCO }\end{array}$ \\
\hline $\begin{array}{l}\text { Wodkowski } \\
2015^{81}\end{array}$ & Canada & 17 & $\begin{array}{l}16 \\
\text { monospecific } \\
\text { anti PM75 } \\
\text { and } 11 \\
\text { anti-PM100 }\end{array}$ & $\begin{array}{l}\text { anti-PM75 } \\
\text { and anti- } \\
\text { PM100 }\end{array}$ & $\begin{array}{l}\text { - Both anti-PM75 and anti-PM100: myositis } \\
\text { - anti-PM75: ILD, calcinosis } \\
\text {. Anti-PM100: calcinosis, better survival }\end{array}$ \\
\hline Liaskos $2017^{89}$ & $\begin{array}{l}\text { Greece, } \\
\text { Germany, USA }\end{array}$ & 19 & $131 \quad \mathrm{~A}$ & $\begin{array}{l}\text { ATA, ACA, a-RNAP III } \\
\quad \text { (RP11, RP155), } \\
\text { anti-fibrillarin, anti-Ku, } \\
\text { anti-NOR90, anti-PM- } \\
\text { Scl100,anti-PM-Scl75 }\end{array}$ & $\begin{array}{l}\text { - } \text { ATA: dcSSc, ILD, PH and ILD-PH, digital ulcers (NS) } \\
\text { - } \text { ACA (anti-CENPB): lcSSc, negatively ILD } \\
\text { · } \text { anti-RP11: male sex } \\
\text { · } \\
\text { - } \quad \text { anti-NOR90: male predominance, ILD } \\
\text { ant: arthritis }\end{array}$ \\
\hline
\end{tabular}

ACA: anticentromere autoantibodies; ANA: antinuclear autoantibodies; a-RNAP: antibodies to RNA polymerase; ATA: antibodies to topoisomerase I; CENP: centromeric protein ; CREST: calcinosis, RP, esophageal involvement, sclerodactyly, telangiectasia; dcSSc: diffuse cutaneous SSc; DLCO: diffusing capacity for carbon monoxide; ESR: erythrocyte sedimentation rate; FVC: forced vital capacity; GAVE: gastric antral vascular ectasia; GFR: glomerular filtration rate; GI: gastrointestinal; ILD: interstitial lung disease; lcSSc: limited cutaneous SSc; MCP: metacarpophalangeal joints; mRSS: modified Rodnan skin score; NS: not significant; PAH: pulmonary arterial hypertension; PH: pulmonary hypertension; PDGFR: platelet-derived growth factor receptor; PM: polymyositis; RNAP: RNA polymerase antibodies; RP: Raynaud phenomenon; sPAP: systolic pulmonary artery pressure; SRC: scleroderma renal crisis; SSc: systemic sclerosis; STROBE: Strengthening the Reporting of Observational Studies in Epidemiology checklist.

system includes a time determinant reflective of the pace of disease, and thus has a prognostic value. Barnett, et al ${ }^{10} \mathrm{empha}-$ sized the importance of assessing the extent of skin involvement within the first year of presentation to place a patient into a specific type. Indeed, type I and II patients had a better prognosis in terms of life expectancy compared to type III. However, only slight difference in survival was found between patients with iSSc and those with ISSc.

Patients with iSSc were found to have variable clinical features and represented a serologically heterogeneous group. It raises the question of iSSc as a distinct variant. Some authors suggested that further subdivision of iSSc might be necessary to identify the subsets with particular patterns of internal organ damage and outcome. Scussel-Lonzetti, et a ${ }^{25}$ divided iSSc into "above elbow" and "below elbow" groups but found them similar with respect to internal organ involvement, mortality, and autoantibody profile. Although the authors supported the concept of an iSSc subset, differentiation was shown only between the LeRoy subsets ("normal + limited" vs "intermediate + diffuse") in terms of heart involvement, disease activity (elevated erythrocyte sedimentation rate [ESR], anemia), and pulmonary fibrosis. The most significant difference in survival rates was found between ISSc and dSSc, whereas the difference between other subsets was absent (ISSc vs iSSc, $P=0.2$ ) or very low (iSSc vs dSSc, 
Table 4. Associations between nailfold capillary patterns and clinical manifestations of SSc.

\begin{tabular}{llcl}
\hline Citation $\quad$ Country & STROBE & $\begin{array}{c}\text { No. of } \\
\text { Patients }\end{array}$ & Classification \\
\hline
\end{tabular}

$\begin{array}{lcccc}\text { Chen } 1984^{91} & \text { USA, China } & 18 & 68 \text { SSc } & \text { Slow and active } \\ \begin{array}{lcccc}\text { Caramaschi } \\ 2007^{92}\end{array} & \text { Italy } & 21 & 103 \text { SSc } & \text { Early, active, late }\end{array}$

$\begin{array}{lcccc}\begin{array}{l}\text { Ingegnoli } \\ 2013^{93}\end{array} & \text { EUSTAR } & 21 & 2754 \text { SSc } & \text { Early, active, late } \\ & & & & \\ \begin{array}{l}\text { Shenavandeh } \\ 2017^{94}\end{array} & \text { Iran } & 19 & 70 \text { SSc } & \begin{array}{c}\text { Normal, early, } \\ \text { active, late, nonspecific }\end{array}\end{array}$

$\begin{array}{lcccc}\text { Cutolo } 2004^{95} & \text { Italy } & 19 & 241 \mathrm{SSc} & \text { Early, active, late } \\ \text { Cutolo } 2016^{96} & \begin{array}{c}\text { Europe, } \\ \text { multicenter }\end{array} & 22 & \begin{array}{c}623 \mathrm{SSc} \text { from } \\ 59 \text { centers } \\ (14 \text { countries })\end{array} & \begin{array}{c}\text { Normal, early, } \\ \text { active, late }\end{array} \\ \text { Bruni } 2015^{97} & \text { Italy } & 17 & \begin{array}{c}110 \mathrm{SSc} \\ \text { Early, active, late }\end{array} & \text { (1) } \\ \text { Smith } 2012^{98} & \text { Italy } & 18 & 66 \mathrm{SSc} & \begin{array}{c}\text { Normal, early, } \\ \text { active, late. }\end{array}\end{array}$

- Slow capillary pattern: ACA

Active: extensive skin involvement and greater visceral involvement (muscle, kidney), more often hypertension

- Severity of skin, lung, heart, and peripheral vascular involvement, as well as homocysteine plasma levels progressively increased across the patterns, from early to late.

- Early and active patterns were more common in $\mathrm{lcSSc}$, whereas a late pattern was more common in dcSSc.

- Late: increased risk of active disease, DUs and moderate-to-severe skin (mRSS $\geq 15$ ), heart, and lung (lowest DLCO and FVC) involvement, risk of ILD

Severity for skin involvement and number of systemic manifestations progressively increased across the patterns.

- Early and active: mild/moderate skin involvement and a low number of disease manifestations

- Late: more severe disease; ATA-positive cases with diffuse cutaneous involvement

- Early: early lcSSc (< 5 yrs) vs early dcSSc (> 3 yrs)

- Late and active: skin telangiectasia, pitting scars, and pulmonary rales compared to those with early pattern

- Late: limitation of the finger-to-palm range of motion, FEV1 $<70 \%$ compared to active and early (only in the early SSc subgroup and lcSSc subtype)

Early and active: $1 \mathrm{cSS} c, \mathrm{ACA}+$

Late: dcSSc, longer duration of RP and SSc, more advanced age, ACAActive and late: ATA

Late: an increased risk of new digital ulcers during a 6-month observation period (OR for late vs normal/early pattern 4.2)

- Early and active: DUs (96\%) compared to patients without a history or present DUs (66\%)

- Early: presence and/or history of DUs

The OR of future severe peripheral vascular and lung involvement at 18-24 months (defined as category $2-4$ DSS per organ) rose steadily throughout the patterns.

$\begin{array}{lcccc}\text { Sulli } 2013^{99} & \text { Belgium, Italy } & 15 & \text { 42 SSc } & \text { Early, active, late } \\ \text { Smith } 2013^{100} & \begin{array}{c}\text { Belgium, } \\ \text { Italian }\end{array} & 17 & 148 & \begin{array}{c}\text { Normal, early, } \\ \text { active, late }\end{array}\end{array}$

- ANA- patients had a slower progression of nailfold microangiopathy characterized by the early pattern.

- Progression to the late pattern was associated with a different autoantibody pattern on IIF (fine-speckled + nucleolar pattern being most prevalent).

- Late: ATA

The OR to develop novel future severe organ involvement (in any of 9 organ systems, defined as category 2-4 per organ of the DSS at 18-24 months) was stronger according to more severe NVC patterns and similar in both cohorts.

ACA: anticentromere autoantibodies; ANA: antinuclear autoantibodies; ATA: antibodies to topoisomerase I; dcSSc: diffuse cutaneous SSc; DLCO: diffusing capacity for carbon monoxide; DSS: disease severity score; DU: digital ulcer; FEV1: forced expiratory volume in 1 second; FVC: forced vital capacity; IIF: indirect immunofluorescence; ILD: interstitial lung disease; lcSSc: limited cutaneous SSc; mRSS: modified Rodnan skin score; NVC: nailfold video capillaroscopy; RP: Raynaud phenomenon; SSc: systemic sclerosis; STROBE: Strengthening the Reporting of Observational Studies in Epidemiology checklist.

$P=0.03)$. ATA positivity was similar between iSSc and dSSc while ACA frequencies gradually decreased from ISSc through iSSc to dSSc (50\%, 34\%, and 3.4\%, respectively). Supporting the LeRoy system, the skin involvement proximal to MCP joints was one of the strong predictors of mortality. In line with those findings, Vayssairat, et a ${ }^{23}$ showed the advantages of LeRoy subset system and disutility of adding iSSc as a subset. When patients with proximal skin thickening were divided into intermediate and truncal subsets, no difference in severity score was found between them.

The patients with calcinosis, RP, esophageal involvement, sclerodactyly, telangiectasia (CREST) syndrome, suspected 


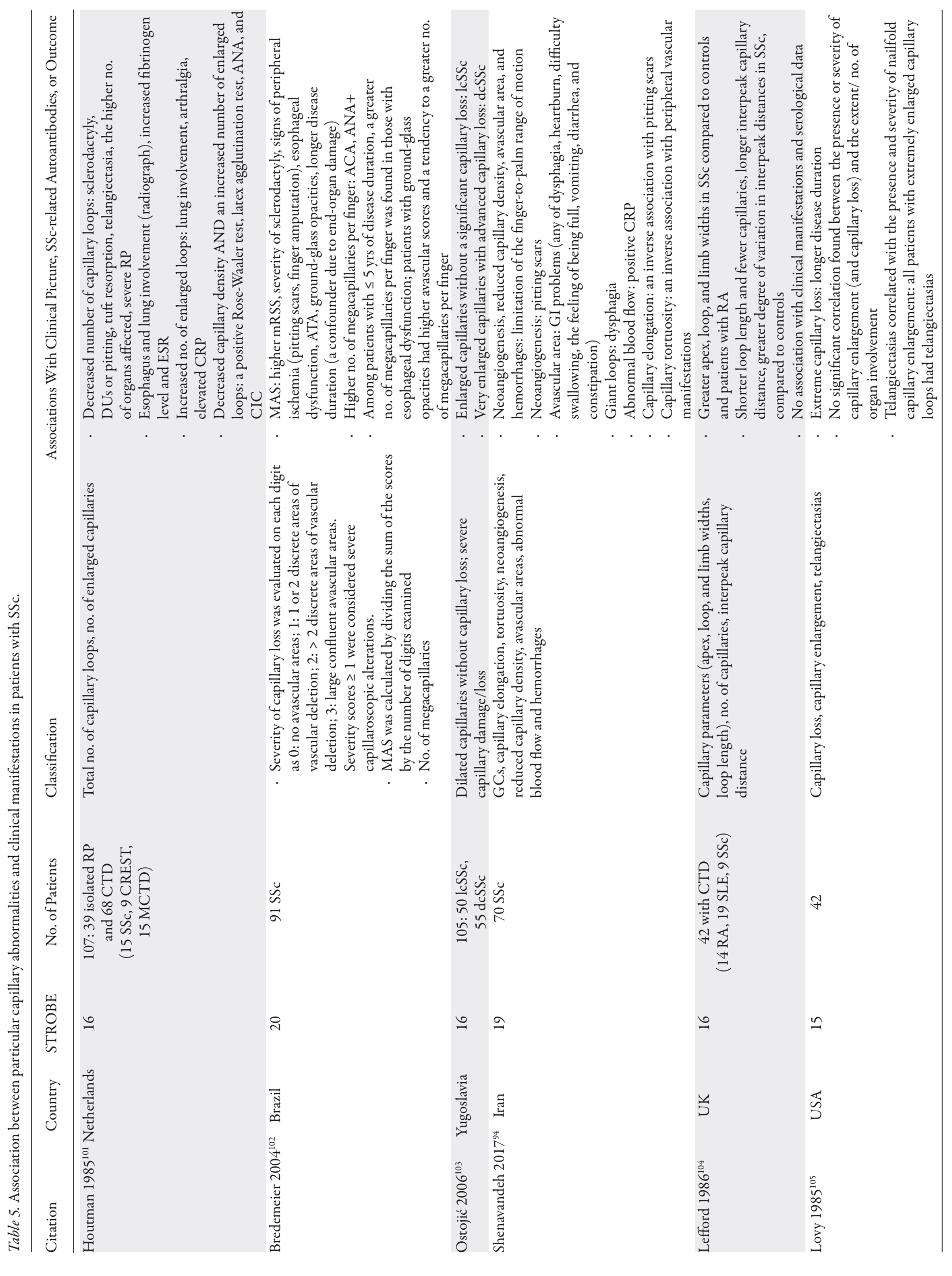




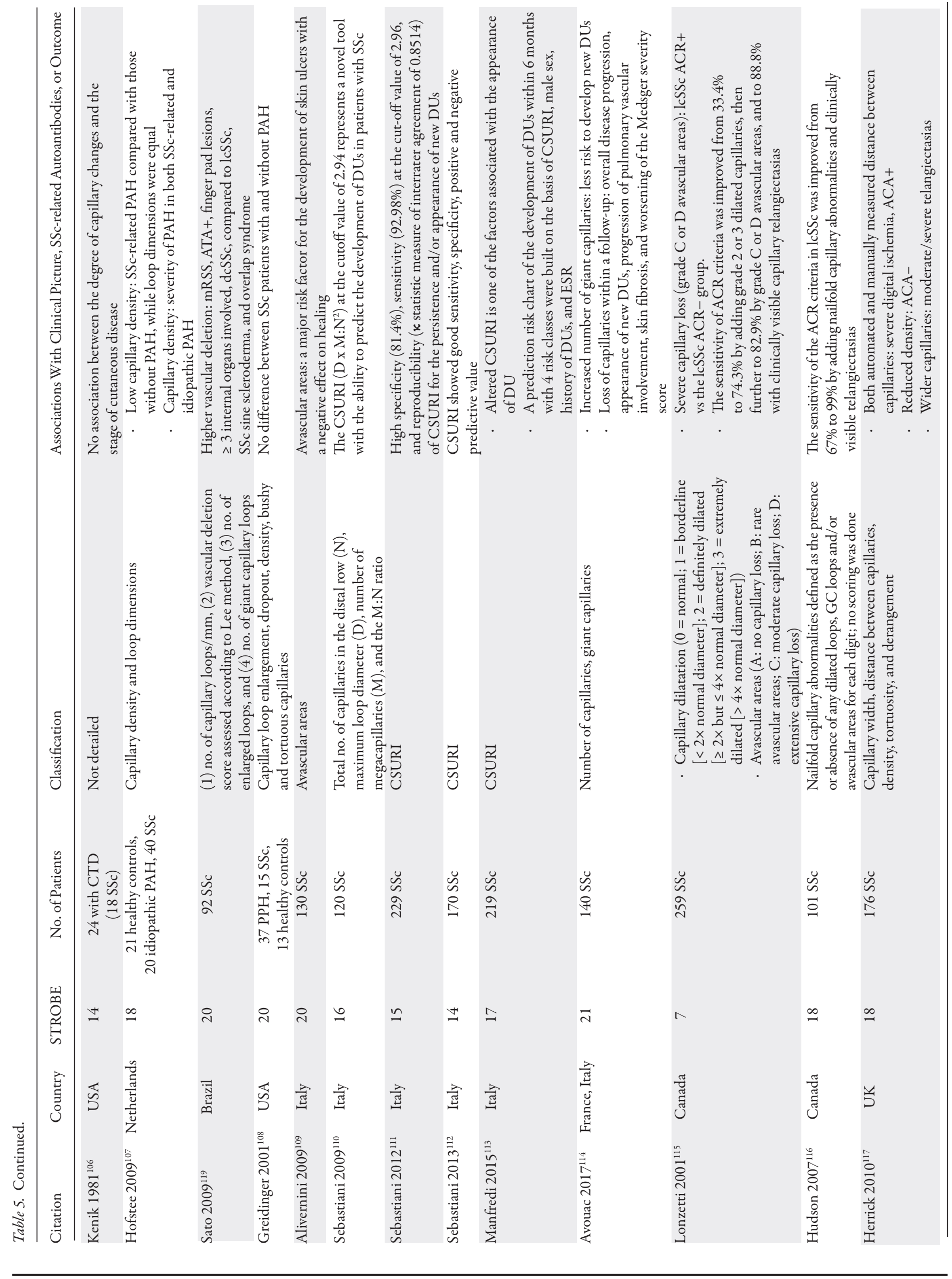




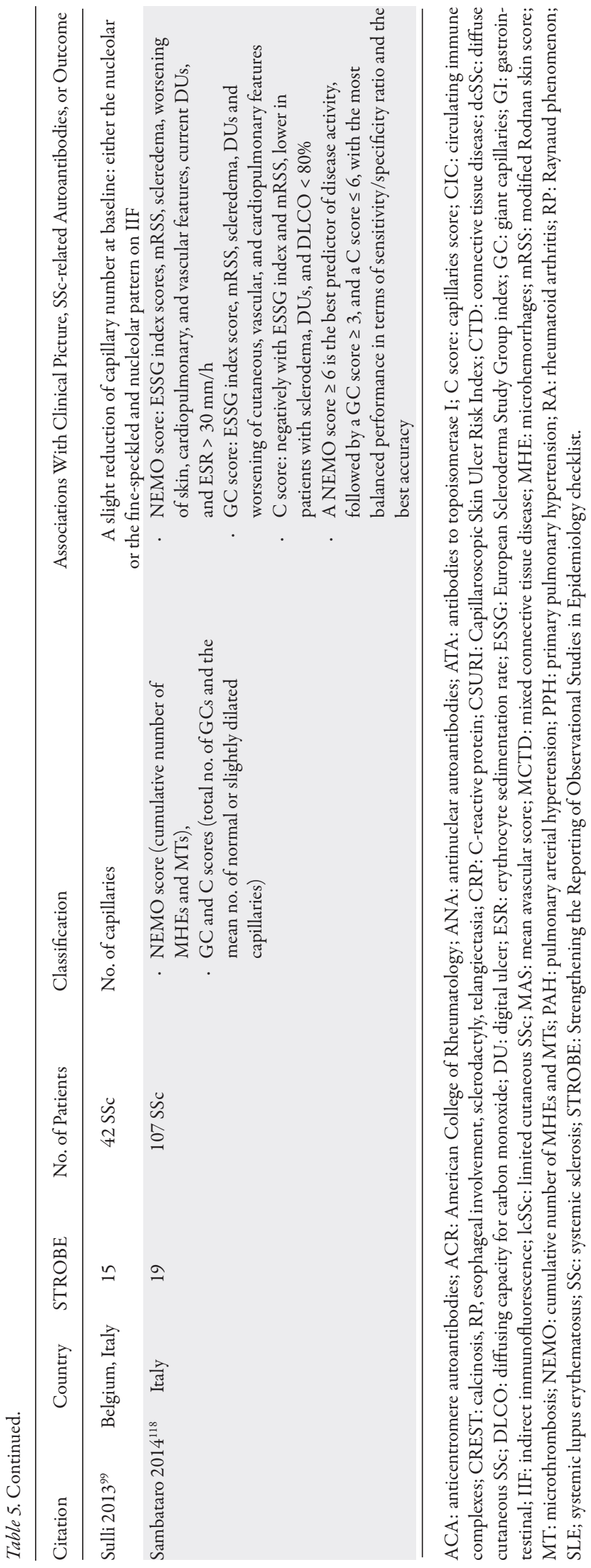

secondary RP, and/or visceral SSc without skin involvement were not acknowledged in the aforementioned 2 classification systems. ${ }^{9,10}$ The recently developed immunoblotting technique to detect SSc-related autoantibodies and nailfold capillary microscopy allows the detection of these probable connective tissue diseases. Expanding the subsets, Maricq, et a ${ }^{2}$ added undifferentiated connective tissue disorder with SSc features, SSc sine scleroderma, and CREST. This classification allows the inclusion of patients who are in earlier stages of their disease.

Boonstra, $e t a l^{27}$ identified 4 clinical subgroups by hierarchical clustering using skin, musculoskeletal, cardiac, pulmonary, and GI manifestations; demographics; and risk assessment using follow-up data. Subgrouping patients allowed the prediction of severity and mortality with 2 subgroups showing higher-than-average 5-year mortality rates: subgroup 1 (male predominance, dcSSc, higher modified Rodnan skin score [mRSS], scleroderma renal crisis (SRC), ATA, less frequent interstitial lung disease [ILD]); and subgroup 2 (female and non-White predominance, more frequent pulmonary arterial hypertension $[\mathrm{PAH}]$, gastric antral vascular ectasia [GAVE], ILD, and lower diffusing lung capacity for carbon monoxide [DLCO] and forced vital capacity [FVC]). Low-risk clusters (subgroups 3 and 4) included patients with lcSSc who were predominantly female, had more frequent GI manifestations (dysphagia, diarrhea, constipation) for both subgroups, as well as peripheral vascular involvement (digital ulcers), ACA, and White predominance for subgroup 3, and less frequent ILD, FVC, and DLCO for subgroup 4. Three subgroups $(1,3$, and 4) were similar to the clusters $(6,3$, and 1 , respectively) in another subclassification system developed by Sobanski, et al as a European Scleroderma Trials and Research Group clustering initiative. ${ }^{37}$ However, 2 main clusters, A and B, in the latter study strongly support the LeRoy $2001^{32}$ subclassification into dcSSc and lcSSc.

SSc subsets based on molecular gene expression profiling. Another approach to classifying patients with SSc into subsets is molecular phenotyping identified through gene expression profiling in tissue samples. Four subsets characterized by distinct molecular pathway signatures have been described and validated in multiple studies: fibroproliferative, inflammatory, normal-like, and limited. ${ }^{38-45,49,121}$ The intrinsic molecular subsets are consistent for each patient, as well as across the different skin biopsy sites, regardless of clinically affected or unaffected status..$^{38,122}$ The subsets are also consistent across the organ systems ${ }^{38,39,42,122}$; however, highly lung-specific innate immune and cell proliferation processes were shown within the immune-fibrotic axis, suggesting that there are gene pairs that are more likely to interact in one tissue than the other (Table 2). ${ }^{123}$

SSc subsets according to SSc-related autoantibodies. The classification system according to serum antibodies is based on the findings of mutually exclusive, SSc-specific autoantibodies that did not change during the course of disease. The autoantibody subsets are distinguished by patterns of cutaneous involvement, specific clinical features, and prognosis (Table 3). SSc-specific autoantibodies were found to be stronger predictors of disease outcome and organ involvement than the extent of skin involvement. ${ }^{27}$ 
The subset of patients with SSc positive for ACA represents a clinically homogenous group with distinct clinical features and seems to have a better prognosis: less severity; less frequent ILD, SRC, inflammatory arthritis, and inflammatory myositis; and patients had lower rates of GI tract involvement, finger ulcers, digital tuft resorption, or finger contractions. The patients are also older at disease onset, predominantly female, and more likely to have limited disease, lower skin scores, telangiectasia and pulmonary hypertension. . $^{10,21,29,51-57,59,61-63,65,69-71,73,74,84,86,89,124}$ ACA status was found to be predictive of the extent of skin involvement over time..$^{59}$ Patients with limited disease who were ACA-negative at baseline were more likely to progress to diffuse disease. ACA-negative patients also had a greater extent of cutaneous involvement, worse survival, and more severe internal organ involvement..$^{29,65}$

Another study supported subdivision of lcSSc into 2 serological subtypes, Th/To-positive and ACA-positive, with different internal organ involvement and outcome. ${ }^{50}$ Compared to the ACA-positive patients, $\mathrm{Th} / \mathrm{To}$-positive patients were younger at disease onset and predominantly male, with less $\mathrm{PAH}$ development, but more ILD ( $38 \%$ vs $4.5 \%$ ). The highest mortality was found in ATA+ and ATA+/ACA- subgroups, while ACA+/ ATA - and $\mathrm{Pm} / \mathrm{Scl}+/ \mathrm{RNA}$ polymerase antibody (RNAP)negative patients were classified as low risk. ${ }^{26}$ Some patients were not within described serological subsets; for example, ACA was commonly found in association with mild skin involvement, but $9 \%$ of dcSSc patients with truncal involvement were positive for ACA. ${ }^{10}$

Caetano, et al described those patients who had a more insidious onset of skin and major organ involvement, a lower incidence of ILD and SRC, and better survival than expected for $\mathrm{dcSS} c$ as a distinct clinical subtype (dcSSc ACA.$+^{70}$ Thus, further subgrouping within each autoantibody profile may be promising from a clinical point of view. Indeed, 2 subgroups of anti-CENPA can explain variable clinical manifestations in an ACA-positive subset. ${ }^{87}$ Subgrouping among patients with SSc positive for anti-RPC155 antibodies (RNAP III large subunit, $155 \mathrm{kDa}$ ) revealed that anti-RPA194 was associated with a lower cancer risk and less severe GI disease, while anti-RNAP I/II/III was associated with SRC..$^{75}$ Therefore, different autoantibody combinations have utility as tools for organ involvement and cancer risk stratification in SSc.

Patterson, $e t a^{B 6}$ reported subgrouping RNAP III-positive patients into 2 clusters; a strongly positive cluster was associated with an increased risk of GAVE, lower risk of esophageal dysmotility, and shorter disease duration. A strong positivity for anti-RNAP III (a higher ELISA index) was associated with the development of SRC. ${ }^{75}$ Although 3 main autoantibodies (ACA, ATA, and anti-RNAP III) have strong mutually exclusive relationships, coexpression of other antibodies are relatively common. ${ }^{86,90,125,126} \mathrm{~A}$ combination of 2 SSc-related autoantibodies was revealed in one-third of patients in the study by Patterson, et al..$^{86}$ Anti-Ro 52 most frequently occurred in combination with other autoantibodies, but coexpressions of ATA with anti-RNAP III $(0.6 \%)$ and ACA (3\%) were also found in a small proportion of patients with SSc. ${ }^{86}$ In cases with coexistence of $\geq 2$ autoantibodies, the autoantibody of highest titer determined the clinical phenotype.

SSc subsets according to nailfold capillary abnormalities. Capillary abnormalities seen on nailfold video capillaroscopy (NVC) can be used to subgroup SSc patients with different clinical manifestations and prognoses. There are 2 classification systems based on the NVC changes (Table 4). First, Maricq, et al ${ }^{127}$ described 2 capillary patterns: "slow" and "active." Slow pattern was characterized by capillary telangiectasias and high number of extremely large (giant) capillary loops with a relatively well-preserved capillary distribution. The main feature of active pattern was moderate-to-extensive capillary loss associated with considerable distortion of the nailfold capillary bed and new blood vessel formation (bushy capillaries). Associations between capillaroscopic findings and disease activity, degree of progression, and prognosis were found. SSc patients with slow pattern predominantly had slowly progressive disease (new symptoms/signs during follow-up were found only in $1 / 11$ patients), longer RP prior to entry, and were ACA-positive, while all patients with active pattern were ACA-negative and half showed disease progression. Capillary loss (active pattern) reflected disease progression that was confirmed in other publications. ${ }^{98,114}$ Ostojic, et al ${ }^{103}$ found that enlarged capillaries without a significant capillary loss (slow pattern) were more frequently seen in lcSSc, whereas giant capillaries (GCs) with advanced capillary loss (active pattern) occurred in dcSSc.

The Maricq NVC classification system has been further subdivided within the active pattern into "active" and "late," whereas slow pattern was renamed as "early" by Cutolo, et al. ${ }^{95,128}$ The principal change was the interpretation of patterns as consecutive phases of progressive obliterative microangiopathy. ${ }^{128}$ Early pattern is characterized by a relatively well-preserved capillary distribution and density with a few enlarged capillaries/GCs, few capillary microhemorrhages, and no evident loss of capillaries. The following moderate loss of capillaries is a sign of the next active phase, with a mildly disturbed architecture of capillaries, frequent GCs and microhemorrhages, capillary derangement, and absent or few ramified capillaries (neoangiogenesis). The capillary changes typical for this phase (hemorrhages and GCs) are closely associated with disease activity. Sambataro, et al showed that NEMO score (cumulative number of microhemorrhages and microthrombosis) $\geq 6$ was the best predictor of disease activity, followed by the GC score (number of GCs) $\geq 3 .{ }^{118}$ The active pattern had more severe disease manifested as extensive skin involvement and greater visceral involvement (muscle, kidney), and patients were ACA-negative in comparison with the early pattern. ${ }^{91}$ In the most advanced phase of SSc microangiopathy, represented by the late NVC pattern, the disorganization of the normal capillary array is generally seen, with severe loss of capillaries and large avascular areas, irregular enlargement of the capillaries, few or absent GCs, microhemorrhages, and ramified/bushy capillaries. Normal NVC pattern is rarely seen in SSc (4-12\%), nearly exclusively in the lcSSc subset. ${ }^{103,129}$ Numerous studies confirmed that patients with more advanced NVC patterns had more severe disease. ${ }^{91,92,93,98,103,127,129}$ Significant capillary loss was 
more common in patients with $\mathrm{lcSS} c$ who met ACR criteria compared to those who did not. ${ }^{115}$

Classifying patients with SSc according to NVC patterns may predict development of a new organ involvement within 1 year. ${ }^{98,100}$ In 2 studies, ${ }^{98,100}$ the odds ratio to develop severe organ involvement (defined as a category 2 or higher in any of the 9 organ systems assessed according to the Medsger Disease Severity Scale, or new PAH or ILD at 18-24 months' follow-up) was stronger according to more severe NVC patterns, adjusting for disease duration, subset, and vasoactive medications. These findings were externally validated in an Italian cohort. ${ }^{100}$ Associations between certain manifestations and NVC patterns are controversial, such as reduced capillary density and PAH. ${ }^{107,108}$ Sample size was sometimes too small to detect possible associations. ${ }^{104}$

All 3 NVC patterns can be observed in both clinical disease subsets ( $\mathrm{lcSSc}$ and $\mathrm{dcSSc})^{128}$; however, early pattern is more common in $1 \mathrm{cSSc}$, especially early $\mathrm{lcSSc}^{93}$ whereas the late pattern is more prevalent in $\mathrm{dcSSc} .{ }^{92,93}$ Classifying patients into NVC subsets is important early in the disease course because capillary loss is a reliable indicator of rapidly progressive early disease. ${ }^{25,94}$ Shenavandeh, et al showed that late pattern in patients with early SSc was associated with severity of finger contractures and significantly reduced pulmonary function, compared to active and early patterns. ${ }^{94}$ Table 4 demonstrates that the reduced number of capillaries typical for active and late patterns was more commonly seen in patients with longer disease duration, higher $\mathrm{mRSS}$, more severe lung (including PAH), GI, and peripheral vascular involvement, a higher number of organs affected, and elevated ESR and C-reactive protein. ${ }^{94,101-103,105,107,109-114,117,118,119}$ The ACR criteria sensitivity may be improved by adding the NVC patterns. ${ }^{115,116}$ More severe NVC patterns (active and late) occurred in patients seropositive for ATA and anti-RNAP III, and negative for ACA. ${ }^{93,95,117,119}$ ANA-negative ${ }^{99}$ and ACA-positive ${ }^{94}$ patients had the most favorable early pattern. However, SSc-related autoantibodies are not directly linked with the development of a distinct SSc NVC pattern (Table 4 and Table 5). ${ }^{129}$

The limitations included small proportions of patients with each NVC pattern (especially early pattern), resulting in limited power to detect statistically significant differences. Some outcomes were omitted from the analysis (i.e., GI involvement and SRC), while others might have been interrelated (i.e., abnormalities in the cardiac measures might be secondary to pulmonary involvement, rather than present as primary cardiac involvement). Further, follow-up duration in the prospective studies varied and was relatively short. Definitions of organ involvement also varied between the studies, which made the comparison of the results difficult.

\section{DISCUSSION}

SSc subset classification is a rapidly evolving field. Our systematic review highlights both the continued importance of skin involvement and the novel role of SSc-specific antibodies, abnormal nailfold capillary patterns, and molecular profiling in assessing patients to determine a subset.
The dcSSc subset comprises patients with rapidly progressive disease who require more aggressive treatment. However, disease progression assessed as severity-duration ratio (early significant visceral and skin involvement) suggests disease activity only in early dcSSc. ${ }^{23,130,131}$ In later stages of disease, patients classified as rapid progressors in the beginning may still have a high disease severity due to the accumulated significant damage, but low disease activity as a result of treatment or spontaneous remission. Some patients with SSc first develop severe skin involvement and/or visceral disease late in the disease course. Thus, the limited/diffuse system loses its predictive value in more advanced disease and should be supplemented with a necessary determination of disease activity and severity when it comes to choosing treatment. With the recent advances in antibody detection, some novel SSc-specific autoantibodies could be added to SSc subset classification autoantibody profiling to the skin involvement while determining a subset.

Based on gene expression profiling, patients with lcSSc can be assigned to the limited, inflammatory, or normal-like subsets, whereas fibroproliferative subset can be seen in patients with dcSSc. The molecular subsets seem to be a universal feature of SSc end-target organ pathology, not affected significantly by heterogeneity of skin involvement within a patient and/or fibroblast heterogeneity in tissues. ${ }^{38,39,122}$ The molecular intrinsic subset assignment could represent a valuable approach for matching patients with SSc to appropriate therapies. Molecular phenotyping may aid personalized medicine by identifying therapies with higher potential for success in each individual patient, as well as to select patients with SSc who will improve naturally as part of their disease course. ${ }^{47}$

Some limitations of subgrouping by molecular phenotyping include the relatively small sample sizes of clinical trials due to the rarity of disease itself, specific inclusion criteria that misrepresents the full spectrum of SSc, lack of controls, and differences in methods of transcript quantification and in the exact list of genes between studies. Moreover, not all therapy- or disease-relevant genes are regulated at the mRNA level. The use of molecular subsetting in clinical practice for individual patients is limited, as paired skin samples from each individual are often not available, analyses are not standardized, and large numbers of samples in a dataset are needed to identify the molecular subset with accuracy. Recently, supervised machine learning algorithms have been developed and may be successfully used to assign single samples to intrinsic gene expression subsets according to predefined criteria. ${ }^{47}$ The method utilizes a multinomial elastic net classifier and an optimized set of genes. Classifier accuracy in that study was proved using concordance of samples $(83.3 \%)$ reporting Cohen $x$ coefficient (0.7391), and was externally validated. Further efforts are needed to explore molecular heterogeneity and intrinsic subsets in other tissues and particularly in peripheral blood, given its accessibility.

Attempts to identify SSc subsets considering SSc-specific autoantibodies have faced a variety of challenges. Boonstra, et al reported that adding autoantibody status to the cluster process resulted in correct classification of patients with ILD, PAH, and SRC. ${ }^{27}$ All high-risk patients were correctly identified by taking 
autoantibodies into account, but the number of patients incorrectly identified as possibly high risk increased significantly (by $66 \%$ ), suggesting limited additional value of autoantibody status for clustering. ${ }^{27}$ The limitations of studies on SSc-specific autoantibodies included underestimation of the number of antigens due to the limitations of the techniques not allowing the identification of membrane proteins, or to a loss of proteins at each step, small sample size, a lack of validation groups, and/or limited generalizability (e.g., SRC is rare in Japanese patients; clinical features in each SSc-related ANA-based subgroup appear to vary among populations of different backgrounds). Feasibility is another consideration, as some autoantibodies are identified by immunoprecipitation, which is not widely used in clinical laboratories, and/or some detection kits are not commercially available. Limitations of classification systems developed by cluster analysis are the exclusion of a significant number of patients due to missing data and/or loss to follow-up that affects the extrapolation of the results. Finally, there have been inconsistent definitions of variables between the studies, a lack of analysis of the potential effect of treatment regimens on survival, and the influence of disease duration on the clustering process.

In conclusion, modern methods to subset SSc include skin involvement, immunologic profile, molecular signatures, visceral involvement, and age. Classifying on the basis of skin involvement, NVC, and autoantibody profile may allow early prediction of internal organ involvement. Molecular subsetting may identify those who are likely to respond to therapy. Longitudinal prospective studies to track subsets are needed to provide insight into disease trajectory, assess their predictive value, and confirm a possible transition between subsets and evolution under treatment.

\section{ACKNOWLEDGMENT}

We are thankful to Melanie Anderson, an information specialist at the University Health Network Library Services, and Keshini Devakandan, a clinical research analyst in the Toronto Scleroderma Program, for their assistance with the literature search.

\section{ONLINE SUPPLEMENT}

Supplementary material accompanies the online version of this article.

\section{REFERENCES}

1. Hinchcliff M, Varga J. Systemic sclerosis/scleroderma: a treatable multisystem disease. Am Fam Physician 2008;78:961-8.

2. Olsen NJ, King LE Jr, Park JH. Muscle abnormalities in scleroderma. Rheum Dis Clin North Am 1996;22:783-96.

3. Steen VD. The lung in systemic sclerosis. J Clin Rheumatol 2005; 11:40-6.

4. Rose S, Young MA, Reynolds JC. Gastrointestinal manifestations of scleroderma. Gastroenterol Clin North Am 1998;27:563-94.

5. Steen VD. Renal involvement in systemic sclerosis. Clin Dermatol 1994; 12:253-8.

6. Turk M, Pope JE. The frequency of scleroderma renal crisis over time: a metaanalysis. J Rheumatol 2016;43:1350-5.

7. Johnson SR, Glaman DD, Schentag CT, Lee P. Quality of life and functional status in systemic sclerosis compared to other rheumatic diseases. J Rheumatol 2006;33:1117-22.

8. van den Hoogen F, Khanna D, Fransen J, Johnson SR, Baron M, Tyndall A, et al. 2013 classification criteria for systemic sclerosis: an American College of Rheumatology/European League Against Rheumatism collaborative initiative. Arthritis Rheum 2013;65:2737-47.

9. LeRoy EC, Black C, Fleischmajer R, Jablonska S, Krieg T, Medsger TA Jr, et al. Scleroderma (systemic sclerosis): classification, subsets and pathogenesis. J Rheumatol 1988;15:202-5.

10. Barnett AJ, Miller MH, Littlejohn GO. A survival study of patients with scleroderma diagnosed over 30 years (1953-1983): the value of a simple cutaneous classification in the early stages of the disease. J Rheumatol 1988;15:276-83.

11. Ferri C, Valentini G, Cozzi F, Sebastiani M, Michelassi C, La Montagna G, et al; Systemic Sclerosis Study Group of the Italian Society of Rheumatology (SIR-GSSSc). Systemic sclerosis: demographic, clinical, and serologic features and survival in 1,012 Italian patients. Medicine 2002;81:139-53.

12. Matucci-Cerinic M, Bellando-Randone S, Lepri G, Bruni C, Guiducci S. Very early versus early disease: the evolving definition of the 'many faces' of systemic sclerosis. Ann Rheum Dis 2013; 72:319-21.

13. Meyer OC, Fertig N, Lucas M, Somogyi N, Medsger TA Jr. Disease subsets, antinuclear antibody profile, and clinical features in 127 French and 247 US adult patients with systemic sclerosis. J Rheumatol 2007;34:104-9.

14. Fertig N, Domsic RT, Rodriguez-Reyna T, Kuwana M, Lucas M, Medsger TA Jr, et al. Anti-U11/U12 RNP antibodies in systemic sclerosis: a new serologic marker associated with pulmonary fibrosis. Arthritis Rheum 2009;61:958-65.

15. Gorlova O, Martin JE, Rueda B, Koeleman BP, Ying J, Teruel M, et al. Identification of novel genetic markers associated with clinical phenotypes of systemic sclerosis through a genome-wide association strategy. PLoS Genet 2011;7:e1002178.

16. van Bon L, Affandi AJ, Broen J, Christmann RB, Marijnissen RJ, Stawski L, et al. Proteome-wide analysis and CXCL4 as a biomarker in systemic sclerosis. N Engl J Med 2014; 370:433-43.

17. Johnson SR, Goek ON, Singh-Grewal D, Vlad SC, Feldman BM, Felson DT, et al. Classification criteria in rheumatic diseases: a review of methodologic properties. Arthritis Rheum 2007; 57:1119-33.

18. Johnson SR, van den Hoogen F, Devakandan K, Matucci-Cerinic M, Pope JE. Systemic sclerosis: to subset or not to subset, that is the question. Eur J Rheumatol 2020;7 Suppl 3:S222-7.

19. Johnson SR, Soowamber ML, Fransen J, Khanna D, Van Den Hoogen F, Baron M, et al. There is a need for new systemic sclerosis subset criteria. A content analytic approach. Scand J Rheumatol 2018;47:62-70.

20. Johnson SR, Feldman BM, Hawker GA. Classification criteria for systemic sclerosis subsets. J Rheumatol 2007;34:1855-63.

21. Ferri C, Bernini L, Cecchetti R, Latorraca A, Marotta G, Pasero G, et al. Cutaneous and serologic subsets of systemic sclerosis. J Rheumatol 1991;18:1826-32.

22. Maricq HR, Valter I. A working classification of scleroderma spectrum disorders: a proposal and the results of testing on a sample of patients. Clin Exp Rheumatol 2004;22:S5-13.

23. Vayssairat M, Baudot N, Abuaf N, Johanet C. Long-term follow-up study of 164 patients with definite systemic sclerosis: classification considerations. Clin Rheumatol 1992;11:356-63.

24. Barnett AJ, Coventry DA. Scleroderma. 1. Clinical features, course of illness and response to treatment in 61 cases. Med J Aust 1969;1:992-1001.

25. Scussel-Lonzetti L, Joyal F, Raynauld JP, Roussin A, Rich E, Goulet JR, et al. Predicting mortality in systemic sclerosis: analysis of a cohort of 309 French Canadian patients with emphasis on features at diagnosis as predictive factors for survival. Medicine 2002;81:154-67. 
26. Simeon CP, Armadans L, Fonollosa V, Vilardell M, Candell J, Tolosa C, et al. Survival prognostic factors and markers of morbidity in Spanish patients with systemic sclerosis. Ann Rheum Dis 1997;56:723-8.

27. Boonstra M, Mertens BJA, Bakker JA, Ninaber MK, Ajmone Marsan N, van der Helm-van Mil AHM, et al. To what extent do autoantibodies help to identify high-risk patients in systemic sclerosis? Clin Exp Rheumatol 2018;36 Suppl 113:109-17.

28. Avouac J, Fransen J, Walker UA, Riccieri V, Smith V, Muller C, et al; EUSTAR Group. Preliminary criteria for the very early diagnosis of systemic sclerosis: results of a Delphi Consensus Study from EULAR Scleroderma Trials and Research Group. Ann Rheum Dis 2011;70:476-81.

29. Giordano M, Valentini G, Migliaresi S, Picillo U, Vatti M. Different antibody patterns and different prognoses in patients with scleroderma with various extent of skin sclerosis. J Rheumatol 1986;13:911-6.

30. Goetz R, Berne MB. The pathophysiology of progressive systemic sclerosis (generalised scleroderma) with special reference to changes in the viscera. Clin Proc 1945;4:337-92.

31. Holzmann H, Sollberg S, Altmeyer P. [Classification of progressive systemic scleroderma]. [Article in German] Hautarzt 1987; 38:253-7.

32. LeRoy EC, Medsger TA Jr. Criteria for the classification of early systemic sclerosis. J Rheumatol 2001;28:1573-6.

33. Masi AT. Classification of systemic sclerosis (scleroderma): relationship of cutaneous subgroups in early disease to outcome and serologic reactivity. J Rheumatol 1988;15:894-8.

34. Rodnan GP, Jablonska S, Medsger TA Jr. Classification and nomenclature of progressive systemic sclerosis. Clin Rheumatic Dis 1979;5:5-13.

35. Winterbauer RH. Multiple telangiectasia, Raynaud's phenomenon, sclerodactyly, and subcutanious calcinosis: a syndrome mimicking hereditary hemorrhagic telangiectasia. Bull Johns Hopkins Hosp 1964;114:361-83.

36. Tuffanelli DL, Winkelmann RK. Diffuse systemic scleroderma. A comparison with acrosclerosis. Ann Intern Med 1962;57:198-203.

37. Sobanski V, Giovannelli J, Allanore Y, Riemekasten G, Airò P, Vettori S, et al; EUSTAR Collaborators. Phenotypes determined by cluster analysis and their survival in the prospective European scleroderma trials and research cohort of patients with systemic sclerosis. Arthritis Rheumatol 2019;71:1553-70.

38. Milano A, Pendergrass SA, Sargent JL, George LK, McCalmont $\mathrm{TH}$, Connolly MK, et al. Molecular subsets in the gene expression signatures of scleroderma skin. PLoS One 2008;3:e2696.

39. Pendergrass SA, Lemaire R, Francis IP, Mahoney JM, Lafyatis R, Whitfield ML. Intrinsic gene expression subsets of diffuse cutaneous systemic sclerosis are stable in serial skin biopsies. J Invest Dermatol 2012;132:1363-73.

40. Hinchcliff M, Huang CC, Wood TA, Matthew Mahoney J, Martyanov V, Bhattacharyya S, et al. Molecular signatures in skin associated with clinical improvement during mycophenolate treatment in systemic sclerosis. J Invest Dermatol 2013;133:1979-89.

41. Mahoney JM, Taroni J, Martyanov V, Wood TA, Greene CS, Pioli PA, et al. Systems level analysis of systemic sclerosis shows a network of immune and profibrotic pathways connected with genetic polymorphisms. PLoS Comput Biol 2015;11:e1004005.

42. Taroni JN, Martyanov V, Huang CC, Mahoney JM, Hirano I, Shetuni B, et al. Molecular characterization of systemic sclerosis esophageal pathology identifies inflammatory and proliferative signatures. Arthritis Res Ther 2015;17:194.

43. Chakravarty EF, Martyanov V, Fiorentino D, Wood TA, Haddon DJ, Jarrell JA, et al. Gene expression changes reflect clinical response in a placebo-controlled randomized trial of abatacept in patients with diffuse cutaneous systemic sclerosis. Arthritis Res Ther 2015;17:159.

44. Gordon JK, Martyanov V, Franks JM, Bernstein EJ, Szymonifka J, Magro C, et al. Belimumab for the treatment of early diffuse systemic sclerosis: results of a randomized, double-blind, placebo-controlled, pilot trial. Arthritis Rheumatol 2018;70:308-16.

45. Taroni JN, Martyanov V, Mahoney JM, Whitfield ML. A functional genomic meta-analysis of clinical trials in systemic sclerosis: toward precision medicine and combination therapy. J Invest Dermatol 2017;137:1033-41.

46. Frost J, Estivill X, Ramsay M, Tikly M. Dysregulation of the Wnt signaling pathway in South African patients with diffuse systemic sclerosis. Clin Rheumatol 2019;38:933-8.

47. Franks JM, Martyanov V, Cai G, Wang Y, Li Z, Wood TA, et al. A machine learning classifier for assigning individual patients with systemic sclerosis to intrinsic molecular subsets. Arthritis Rheumatol 2019;71:1701-10.

48. van der Kroef M, van den Hoogen LL, Mertens JS, Blokland SLM, Haskett S, Devaprasad A, et al. Cytometry by time of flight identifies distinct signatures in patients with systemic sclerosis, systemic lupus erythematosus and Sjögrens syndrome. Eur J Immunol 2020;50:119-29.

49. Martyanov V, Kim GJ, Hayes W, Du S, Ganguly BJ, Sy O, et al. Novel lung imaging biomarkers and skin gene expression subsetting in dasatinib treatment of systemic sclerosis-associated interstitial lung disease. PLoS One 2017;12:e0187580.

50. Ceribelli A, Cavazzana I, Franceschini F, Airò P, Tincani A, Cattaneo R, et al. Anti-Th/To are common antinucleolar autoantibodies in Italian patients with scleroderma. J Rheumatol 2010;37:2071-5.

51. Gliddon AE, Doré CJ, Dunphy J, Betteridge Z, McHugh NJ, Maddison PJ, et al; QUINS Trial Study Group. Antinuclear antibodies and clinical associations in a British cohort with limited cutaneous systemic sclerosis. J Rheumatol 2011;38:702-5.

52. Falkner D, Wilson J, Fertig N, Clawson K, Medsger TA Jr, Morel PA. Studies of HLA-DR and DQ alleles in systemic sclerosis patients with autoantibodies to RNA polymerases and U3-RNP (fibrillarin). J Rheumatol 2000;27:1196-202.

53. Graf SW, Hakendorf P, Lester S, Patterson K, Walker JG, Smith MD, et al. South Australian Scleroderma Register: autoantibodies as predictive biomarkers of phenotype and outcome. Int J Rheum Dis 2012;15:102-9.

54. Hamaguchi Y, Hasegawa M, Fujimoto M, Matsushita T, Komura $\mathrm{K}$, Kaji K, et al. The clinical relevance of serum antinuclear antibodies in Japanese patients with systemic sclerosis. Br J Dermatol 2008; 158:487-95.

55. Hanke K, Becker MO, Brueckner CS, Meyer W, Janssen A, Schlumberger W, et al. Anticentromere-A and anticentromere-B antibodies show high concordance and similar clinical associations in patients with systemic sclerosis. J Rheumatol 2010;37:2548-52.

56. Harvey GR, Butts S, Rands AL, Patel Y, McHugh NJ. Clinical and serological associations with anti-RNA polymerase antibodies in systemic sclerosis. Clin Exp Immunol 1999;117:395-402.

57. Hesselstrand R, Scheja A, Shen GQ, Wiik A, Akesson A. The association of antinuclear antibodies with organ involvement and survival in systemic sclerosis. Rheumatology 2003;42:534-40.

58. Song G, Hu C, Zhu H, Wang L, Zhang F, Li Y, et al. New centromere autoantigens identified in systemic sclerosis using centromere protein microarrays. J Rheumatol 2013;40:461-8.

59. Hudson M, Mahler M, Pope J, You D, Tatibouet S, Steele R, et al. Clinical correlates of CENP-A and CENP-B antibodies in a large cohort of patients with systemic sclerosis. J Rheumatol 2012; 39:787-94. 
60. Kuwana M, Okano Y, Pandey JP, Silver RM, Fertig N, Medsger TA Jr. Enzyme-linked immunosorbent assay for detection of anti-RNA polymerase III antibody: analytical accuracy and clinical associations in systemic sclerosis. Arthritis Rheum 2005;52:2425-32.

61. McCarty GA, Rice JR, Bembe ML, Barada FA Jr. Anticentromere antibody. Clinical correlations and association with favorable prognosis in patients with scleroderma variants. Arthritis Rheum 1983;26:1-7.

62. Vázquez-Abad D, Wallace S, Senécal JL, Joyal F, Roussin A, Earnshaw WC, et al. Anticentromere autoantibodies. Evaluation of an ELISA using recombinant fusion protein CENP-B as antigen. Arthritis Rheum 1994;37:248-52.

63. Wu R, Shovman O, Zhang Y, Gilburd B, Zandman-Goddard G, Shoenfeld $\mathrm{Y}$. Increased prevalence of anti-third generation cyclic citrullinated peptide antibodies in patients with rheumatoid arthritis and CREST syndrome. Clin Rev Allergy Immunol 2007;32:47-56.

64. Santiago M, Baron M, Hudson M, Burlingame RW, Fritzler MJ. Antibodies to RNA polymerase III in systemic sclerosis detected by ELISA. J Rheumatol 2007;34:1528-34.

65. Salazar GA, Assassi S, Wigley F, Hummers L, Varga J, Hinchcliff $\mathrm{M}$, et al. Antinuclear antibody-negative systemic sclerosis. Semin Arthritis Rheum 2015;44:680-6.

66. Satoh T, Ishikawa O, Ihn H, Endo H, Kawaguchi Y, Sasaki T, et al. Clinical usefulness of anti-RNA polymerase III antibody measurement by enzyme-linked immunosorbent assay. Rheumatology 2009;48:1570-4.

67. Sato S, Hasegawa M, Nagaoka T, Takamatsu Y, Yazawa N, Ihn H, et al. Autoantibodies against calpastatin in sera from patients with systemic sclerosis. J Rheumatol 1998;25:2135-9.

68. Simon D, Czömpöly T, Berki T, Minier T, Peti A, Tóth E, et al. Naturally occurring and disease-associated auto-antibodies against topoisomerase I: a fine epitope mapping study in systemic sclerosis and systemic lupus erythematosus. Int Immunol 2009;21:415-22.

69. Iniesta Arandia N, Simeón-Aznar CP, Guillén Del Castillo A, Colunga Argüelles D, Rubio-Rivas M, Trapiella Martínez L, et al; RESCLE investigators, Autoimmune Diseases Study Group (GEAS). Influence of antibody profile in clinical features and prognosis in a cohort of Spanish patients with systemic sclerosis. Clin Exp Rheumatol 2017;35 Suppl 106:98-105.

70. Caetano J, Nihtyanova SI, Harvey J, Denton CP, Ong VH. Distinctive clinical phenotype of anti-centromere antibody-positive diffuse systemic sclerosis. Rheumatol Adv Pract 2018;2:rky002.

71. Caramaschi P, Tonolli E, Biasi D, Caimmi C, Pieropan S, Dal Forno I, et al. Antinuclear autoantibody profile in systemic sclerosis patients who are negative for anticentromere and anti-topoisomerase I specificities. Joint Bone Spine 2015;82:209-10.

72. Coppo P, Henry-Dessailly I, Rochette J, Lok C, Buendia B, Lassoued $\mathrm{K}$. Clinical significance of autoantibodies to the pericentromeric heterochromatin protein 1a protein. Eur J Intern Med 2013; 24:868-71.

73. Igusa T, Hummers LK, Visvanathan K, Richardson C, Wigley FM, Casciola-Rosen L, et al. Autoantibodies and scleroderma phenotype define subgroups at high-risk and low-risk for cancer. Ann Rheum Dis 2018;77:1179-86.

74. Foocharoen C, Watcharenwong P, Netwijitpan S, Mahakkanukrauh A, Suwannaroj S, Nanagara R. Relevance of clinical and autoantibody profiles in systemic sclerosis among Thais. Int $\mathrm{J}$ Rheum Dis 2017;20:1572-81.

75. Hamaguchi Y, Kodera M, Matsushita T, Hasegawa M, Inaba Y, Usuda T, et al. Clinical and immunologic predictors of scleroderma renal crisis in Japanese systemic sclerosis patients with anti-RNA polymerase III autoantibodies. Arthritis Rheumatol 2015; 67:1045-52.

76. Haddon DJ, Wand HE, Jarrell JA, Spiera RF, Utz PJ, Gordon JK, et al. Proteomic analysis of sera from individuals with diffuse cutaneous systemic sclerosis reveals a multianalyte signature associated with clinical improvement during imatinib mesylate treatment. J Rheumatol 2017;44:631-8.

77. Foocharoen C, Suwannachat P, Netwijitpan S, Mahakkanukrauh A, Suwannaroj S, Nanagara R, et al. Clinical differences between Thai systemic sclerosis patients with positive versus negative anti-topoisomerase I. Int J Rheum Dis 2016;19:312-20.

78. Hoa S, Hudson M, Troyanov Y, Proudman S, Walker J, Stevens W, et al; Canadian Scleroderma Research Group (CSRG); Australian Scleroderma Interest Group (ASIG); Genetics versus Environment in Scleroderma Outcome Study (GENISOS). Single-specificity anti-Ku antibodies in an international cohort of 2140 systemic sclerosis subjects: clinical associations. Medicine 2016;95:e4713.

79. Terras S, Hartenstein H, Höxtermann S, Gambichler T, Kreuter A. RNA polymerase III autoantibodies may indicate renal and more severe skin involvement in systemic sclerosis. Int J Dermatol 2016;55:882-5.

80. Perosa F, Favoino E, Cuomo G, Digiglio L, Dammacco F, Prete M, et al. Clinical correlates of a subset of anti-CENP-A antibodies cross-reacting with FOXE3p53-62 in systemic sclerosis. Arthritis Res Ther 2013;15:R72.

81. Wodkowski M, Hudson M, Proudman S, Walker J, Stevens W, Nikpour M, et al; Canadian Scleroderma Research Group (CSRG); Australian Scleroderma Cohort Study (ASCS); Genetics versus Environment in Scleroderma Outcome Study (GENISOS). Monospecific anti-Ro52/TRIM21 antibodies in a tri-nation cohort of 1574 systemic sclerosis subjects: evidence of an association with interstitial lung disease and worse survival. Clin Exp Rheumatol 2015;33:S131-5.

82. Shah AA, Rosen A, Hummers L, Wigley F, Casciola-Rosen L. Close temporal relationship between onset of cancer and scleroderma in patients with RNA polymerase I/III antibodies. Arthritis Rheum 2010;62:2787-95.

83. Sánchez-Montalvá A, Fernandez-Luque A, Simeón CP, Fonollosa-Plà V, Marín A, Guillén A, et al. Anti-SSA/Ro52 autoantibodies in scleroderma: results of an observational, cross-sectional study. Clin Exp Rheumatol 2014;32:S177-82.

84. Shah AA, Laiho M, Rosen A, Casciola-Rosen L. Protective effect against cancer of antibodies to the large subunits of both RNA polymerases I and III in scleroderma. Arthritis Rheumatol 2019;71:1571-79.

85. Shayakhmetova RU, Ananyeva LP. Mixed connective tissue disease. Mod Rheumatol 2019;13:11-18.

86. Patterson KA, Roberts-Thomson PJ, Lester S, Tan JA, Hakendorf P, Rischmueller M, et al. Interpretation of an extended autoantibody profile in a well-characterized Australian systemic sclerosis (scleroderma) cohort using principal components analysis. Arthritis Rheumatol 2015;67:3234-44.

87. Perosa F, Favoino E, Favia IE, Vettori S, Prete M, Corrado A, et al. Subspecificities of anticentromeric protein A antibodies identify systemic sclerosis patients at higher risk of pulmonary vascular disease. Medicine 2016;95:e3931.

88. Wuttge DM, Carlsen AL, Teku G, Steen SO, Wildt M, Vihinen M, et al. Specific autoantibody profiles and disease subgroups correlate with circulating micro-RNA in systemic sclerosis. Rheumatology 2015;54:2100-7.

89. Liaskos C, Marou E, Simopoulou T, Barmakoudi M, Efthymiou G, Scheper T, et al. Disease-related autoantibody profile in patients with systemic sclerosis. Autoimmunity 2017;50:414-21.

90. Wodkowski M, Hudson M, Proudman S, Walker J, Stevens W, Nikpour M, et al. Clinical correlates of monospecific anti-PM75 
and anti-PM100 antibodies in a tri-nation cohort of 1574 systemic sclerosis subjects. Autoimmunity 2015;48:542-51.

91. Chen ZY, Silver RM, Ainsworth SK, Dobson RL, Rust P, Maricq HR. Association between fluorescent antinuclear antibodies, capillary patterns, and clinical features in scleroderma spectrum disorders. Am J Med 1984;77:812-22.

92. Caramaschi P, Canestrini S, Martinelli N, Volpe A, Pieropan S, Ferrari M, et al. Scleroderma patients nailfold videocapillaroscopic patterns are associated with disease subset and disease severity. Rheumatology 2007;46:1566-69.

93. Ingegnoli F, Ardoino I, Boracchi P, Cutolo M; EUSTAR co-authors. Nailfold capillaroscopy in systemic sclerosis: data from the EULAR scleroderma trials and research (EUSTAR) database. Microvasc Res 2013;89:122-128.

94. Shenavandeh S, Haghighi MY, Nazarinia MA. Nailfold digital capillaroscopic findings in patients with diffuse and limited cutaneous systemic sclerosis. Reumatologia 2017;55:15-23.

95. Cutolo M, Pizzorni C, Tuccio M, Burroni A, Craviotto C, Basso M, et al. Nailfold videocapillaroscopic patterns and serum autoantibodies in systemic sclerosis. Rheumatology 2004;43:719-26.

96. Cutolo M, Herrick AL, Distler O, Becker MO, Beltran E, Carpentier P, et al; CAP Study Investigators. Nailfold videocapillaroscopic features and other clinical risk factors for digital ulcers in systemic sclerosis: a multicenter, prospective cohort study. Arthritis Rheumatol 2016;68:2527-39.

97. Bruni C, Guiducci S, Bellando-Randone S, Lepri G, Braschi F, Fiori G, et al. Digital ulcers as a sentinel sign for early internal organ involvement in very early systemic sclerosis. Rheumatology 2015;54:72-6.

98. Smith V, Decuman S, Sulli A, Bonroy C, Piettte Y, Deschepper E, et al. Do worsening scleroderma capillaroscopic patterns predict future severe organ involvement? A pilot study. Ann Rheum Dis 2012;71:1636-9.

99. Sulli A, Ruaro B, Smith V, Pizzorni C, Zampogna G, Gallo M, et al. Progression of nailfold microvascular damage and antinuclear antibody pattern in systemic sclerosis. J Rheumatol 2013;40:634-9.

100. Smith V, Riccieri V, Pizzorni C, Decuman S, Deschepper E, Bonroy C, et al. Nailfold capillaroscopy for prediction of novel future severe organ involvement in systemic sclerosis. J Rheumatol 2013; 40:2023-8.

101. Houtman PM, Kallenberg CG, Wouda AA, The TH. Decreased nailfold capillary density in Raynaud's phenomenon: a reflection of immunologically mediated local and systemic vascular disease? Ann Rheum Dis 1985;44:603-9.

102. Bredemeier M, Xavier RM, Capobianco KG, Restelli VG, Rohde LE, Pinotti AF, et al. Nailfold capillary microscopy can suggest pulmonary disease activity in systemic sclerosis. J Rheumatol 2004;31:286-94.

103. Ostojić P, Damjanov N. Different clinical features in patients with limited and diffuse cutaneous systemic sclerosis. Clin Rheumatol 2006;25:453-7.

104. Lefford F, Edwards JC. Nailfold capillary microscopy in connective tissue disease: a quantitative morphological analysis. Ann Rheum Dis 1986; 45:741-9.

105. Lovy M, MacCarter D, Steigerwald JC. Relationship between nailfold capillary abnormalities and organ involvement in systemic sclerosis. Arthritis Rheum 1985;28:496-501.

106. Kenik JG, Maricq HR, Bole GG. Blind evaluation of the diagnostic specificity of nailfold capillary microscopy in the connective tissue diseases. Arthritis Rheum 1981;24:885-91.

107. Hofstee HM, Vonk Noordegraaf A, Voskuyl AE, Dijkmans BA, Postmus PE, Smulders YM, et al. Nailfold capillary density is associated with the presence and severity of pulmonary arterial hypertension in systemic sclerosis. Ann Rheum Dis 2009;68:191-5.
108. Greidinger EL, Gaine SP, Wise RA, Boling C, Housten-Harris T, Wigley FM. Primary pulmonary hypertension is not associated with scleroderma-like changes in nailfold capillaries. Chest 2001;120:796-800.

109. Alivernini S, De Santis M, Tolusso B, Mannocci A, Bosello SL, Peluso G, et al. Skin ulcers in systemic sclerosis: determinants of presence and predictive factors of healing. J Am Acad Dermatol 2009;60:426-35.

110. Sebastiani M, Manfredi A, Colaci M, D’Amico R, Malagoli V, Giuggioli D, et al. Capillaroscopic skin ulcer risk index: a new prognostic tool for digital skin ulcer development in systemic sclerosis patients. Arthritis Rheum 2009;61:688-94.

111. Sebastiani M, Manfredi A, Vukatana G, Moscatelli S, Riato L, Bocci $\mathrm{M}$, et al. Predictive role of capillaroscopic skin ulcer risk index in systemic sclerosis: a multicentre validation study. Ann Rheum Dis 2012;71:67-70.

112. Sebastiani M, Manfredi A, Lo Monaco A, Praino E, Riccieri V, Grattagliano V, et al. Capillaroscopic Skin Ulcers Risk Index (CSURI) calculated with different videocapillaroscopy devices: how its predictive values change. Clin Exp Rheumatol 2013;31:115-7.

113. Manfredi A, Sebastiani M, Carraro V, Iudici M, Bocci M, Vukatana $\mathrm{G}$, et al. Prediction risk chart for scleroderma digital ulcers: a composite predictive model based on capillaroscopic, demographic and clinico-serological parameters. Clin Hemorheol Microcirc 2015;59:133-43.

114. Avouac J, Lepri G, Smith V, Toniolo E, Hurabielle C, Vallet A, et al. Sequential nailfold videocapillaroscopy examinations have responsiveness to detect organ progression in systemic sclerosis. Semin Arthritis Rheum 2017;47:86-94.

115. Lonzetti LS, Joyal F, Raynauld JP, Roussin A, Goulet JR, Rich E, et al. Updating the American College of Rheumatology preliminary classification criteria for systemic sclerosis: addition of severe nailfold capillaroscopy abnormalities markedly increases the sensitivity for limited scleroderma. Arthritis Rheum 2001;44:735-6.

116. Hudson M, Taillefer S, Steele R, Dunne J, Johnson SR, Jones $\mathrm{N}$, et al. Improving the sensitivity of the American College of Rheumatology classification criteria for systemic sclerosis. Clin Exp Rheumatol 2007;25:754-7.

117. Herrick AL, Moore TL, Murray AK, Whidby N, Manning JB, Bhushan M, et al. Nail-fold capillary abnormalities are associated with anti-centromere antibody and severity of digital ischaemia. Rheumatology 2010;49:1776-82.

118. Sambataro D, Sambataro G, Zaccara E, Maglione W, Polosa R, Afeltra AM, et al. Nailfold videocapillaroscopy micro-haemorrhage and giant capillary counting as an accurate approach for a steady state definition of disease activity in systemic sclerosis. Arthritis Res Ther 2014;16:462.

119. Sato LT, Kayser C, Andrade LE. Nailfold capillaroscopy abnormalities correlate with cutaneous and visceral involvement in systemic sclerosis patients. Acta Reumatol Port 2009;34:219-27.

120. von Elm E, Altman DG, Egger M, Pocock SJ, Gøtzsche PC, Vandenbroucke JP, et al; STROBE Initiative. The Strengthening the Reporting of Observational Studies in Epidemiology (STROBE) statement: guidelines for reporting observational studies. Lancet 2007;370:1453-7.

121. Mak AC, Tang PL, Cleveland C, Smith MH, Kari Connolly M, Katsumoto TR, et al. Brief report: whole-exome sequencing for identification of potential causal variants for diffuse cutaneous systemic sclerosis. Arthritis Rheumatol 2016;68:2257-62.

122. Whitfield ML, Finlay DR, Murray JI, Troyanskaya OG, Chi JT, Pergamenschikov A, et al. Systemic and cell type-specific gene expression patterns in scleroderma skin. Proc Natl Acad Sci U S A 2003;100:12319-24. 
123. Taroni JN, Greene CS, Martyanov V, Wood TA, Christmann RB, Farber HW, et al. A novel multi-network approach reveals tissue-specific cellular modulators of fibrosis in systemic sclerosis. Genome Med 2017;9:27.

124. Lima HC, Muller C, Paiva E. A study of systemic scleroderma in the southern region of Brazil: cutaneous manifestations and clinical and laboratory marker association [abstract]. J Am Acad Dermatol 60:AB63.

125. Maes L, Blockmans D, Verschueren P, Westhovens R, De Beéck KO, Vermeersch P, et al. Anti-PM/Scl-100 and anti-RNA-polymerase III antibodies in scleroderma. Clin Chim Acta 2010;411:965-71.

126. Villalta D, Imbastaro T, Di Giovanni S, Lauriti C, Gabini M, Turi $\mathrm{MC}$, et al. Diagnostic accuracy and predictive value of extended autoantibody profile in systemic sclerosis. Autoimmun Rev 2012;12:114-20.

127. Maricq HR, Harper FE, Khan MM, Tan EM, LeRoy EC. Microvascular abnormalities as possible predictors of disease subsets in Raynaud phenomenon and early connective tissue disease. Clin Exp Rheumatol 1983;1:195-205.
128. Cutolo M, Sulli A, Pizzorni C, Accardo S. Nailfold videocapillaroscopy assessment of microvascular damage in systemic sclerosis. J Rheumatol 2000;27:155-60.

129. Markusse IM, Meijs J, de Boer B, Bakker JA, Schippers HPC, Schouffoer AA, et al. Predicting cardiopulmonary involvement in patients with systemic sclerosis: complementary value of nailfold videocapillaroscopy patterns and disease-specific autoantibodies. Rheumatology 2017;56:1081-8.

130. Clements PJ, Hurwitz EL, Wong WK, Seibold JR, Mayes M, White $\mathrm{B}$, et al. Skin thickness score as a predictor and correlate of outcome in systemic sclerosis: high-dose versus low-dose penicillamine trial. Arthritis Rheum 2000;43:2445-54.

131. Hanitsch LG, Burmester GR, Witt C, Hunzelmann N, Genth E, Krieg T, et al. Skin sclerosis is only of limited value to identify SSc patients with severe manifestations--an analysis of a distinct patient subgroup of the German Systemic Sclerosis Network (DNSS) Register. Rheumatology 2009;48:70-3. 